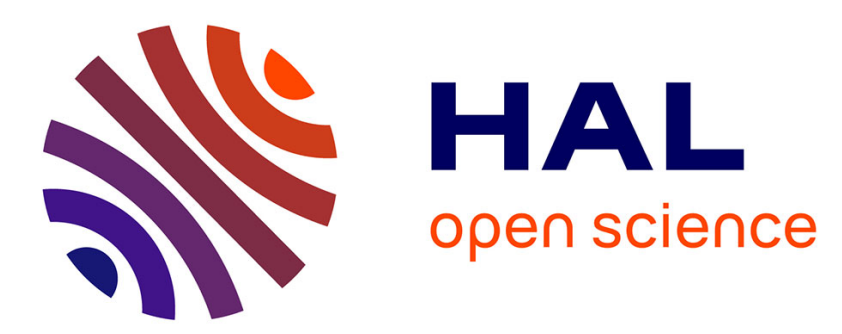

\title{
Artificial equilibrium points for a generalized sail in the circular restricted three-body problem
}

Generoso Aliasi, Giovanni Mengali, Alessandro A. Quarta

\section{To cite this version:}

Generoso Aliasi, Giovanni Mengali, Alessandro A. Quarta. Artificial equilibrium points for a generalized sail in the circular restricted three-body problem. Celestial Mechanics and Dynamical Astronomy, 2011, 110 (4), pp.343-368. 10.1007/s10569-011-9366-y . hal-00659047

\section{HAL Id: hal-00659047 https://hal.science/hal-00659047}

Submitted on 12 Jan 2012

HAL is a multi-disciplinary open access archive for the deposit and dissemination of scientific research documents, whether they are published or not. The documents may come from teaching and research institutions in France or abroad, or from public or private research centers.
L'archive ouverte pluridisciplinaire HAL, est destinée au dépôt et à la diffusion de documents scientifiques de niveau recherche, publiés ou non, émanant des établissements d'enseignement et de recherche français ou étrangers, des laboratoires publics ou privés. 


\title{
Artificial Equilibrium Points for a Generalized Sail in the Circular Restricted Three-Body Problem
}

\author{
by \\ Generoso Aliasi, Giovanni Mengali \\ and \\ Alessandro A. Quarta \\ Corresponding author: \\ Giovanni Mengali \\ University of Pisa, \\ Dipartimento di Ingegneria Aerospaziale, \\ Via G. Caruso 8, I-56122 Pisa, Italy \\ tel: $+39-050-2217220$ \\ fax: $+39-050-2217244$ \\ Email: g.mengali@ing.unipi.it
}

A Paper submitted to 
Revised version \# 3

27th June 2011 
Noname manuscript No.

(will be inserted by the editor)

\title{
Artificial Equilibrium Points for a Generalized Sail in the Circular Restricted Three-Body Problem
}

\author{
Generoso Aliasi · Giovanni Mengali • Alessandro A. Quarta
}

the date of receipt and acceptance should be inserted later

Keywords Artificial Lagrangian Equilibrium points · Radial propulsive acceleration · Generalized sail

\begin{abstract}
This paper introduces a new approach to the study of artificial equilibrium points in the circular restricted three-body problem for propulsion systems with continuous and purely radial thrust. The propulsion system is described by means of a general mathematical model that encompasses the behavior of different systems like a solar sail, a magnetic sail and an electric sail. The proposed model is based on the choice of a coefficient related to the propulsion type and a performance parameter that quantifies the system technological complexity. The propulsion system is therefore referred to as generalized sail. The existence of artificial equilibrium points for a generalized sail is investigated. It is shown that three different families of equilibrium points exist, and their characteristic locus is described geometrically by varying the value of the performance parameter. The linear stability of the artificial points is also discussed.
\end{abstract}

G. Aliasi · G. Mengali · A. A. Quarta

Dipartimento di Ingegneria Aerospaziale, University of Pisa, Via G. Caruso 8, I-56122 Pisa, Italy

G. Aliasi, E-mail: g.aliasi@dia.unipi.it

G. Mengali (corresponding author), E-mail: g.mengali@ing.unipi.it

A. A. Quarta, E-mail: a.quarta@ing.unipi.it 


\section{Introduction}

The Lagrangian points of the Circular Restricted Three-Body Problem (CR3BP) are known to be the five positions in an orbital system rotating with the two massive bodies where a small object (e.g., a spacecraft), affected only by gravity (and centripetal forces), can theoretically be stationary relative to the two larger objects. These points are the only five positions in space where the small object, if placed there, would maintain its position relative to the two massive bodies. If however the object is equipped with a suitable propulsion system, capable of balancing the gravitational pull of the two massive bodies, other equilibrium points can be created allowing the third body to be stationary with respect to the first two bodies. According to Dusek (1965) these new points are usually referred to as Artificial Equilibrium (or Lagrangian) Points (AEPs).

The problem of describing the location of AEPs and of investigating their stability properties has been addressed by several authors. In particular Perezhogin (1976); Kunitsyn and Perezhogin (1978); Schuerman (1980); Simmons et al. (1985); Perezhogin and Tureshbaev (1989) investigated the effects of the thrust due to the radiation pressure on the CR3BP and showed that seven equilibrium points exist if one of the massive bodies is luminous. This problem is equivalent to consider a photonic (or solar) sail, whose thrust direction is fixed and radial with respect to the luminous body. Subsequently, different studies regarding the use of solar sails (McInnes et al., 1994; McInnes, 1999a; Baig and McInnes, 2008; Bookless and McInnes, 2008; Waters and McInnes, 2008) or low-thrust systems (Morimoto et al., 2007) have been carried out, which proved the existence of infinite equilibrium surfaces depending on the magnitude of the propulsive acceleration. However, only a subset of the potentially achievable AEPs turns out to be stable and, as such, could not be exploited by a spacecraft without the use of a suitable control system. The topology of such subset of stable AEPs is strictly dependent on the propulsion system type employed by the spacecraft. In fact, as was recently pointed out by Bombardelli and Peláez (2011), if the available propulsive acceleration is low, the stable AEPs are confined to a very restricted region around the classical Lagrange points.

In this paper a general approach to the CR3BP with a continuous and purely radial thrust (with respect to the more massive body) is discussed. The concept of generalized sail is introduced, in such a way that different continuous propulsion systems can be described within a single mathematical model, 
depending on the choice of a coefficients that defines the propulsion type and a suitable performance parameter that quantifies the system technological complexity. This model allows one to investigate, in an unified framework, the position of the AEPs as a function of the performance parameter and to check their linear stability. In this context the assumption of purely radial thrust is particularly significant as it is representative of a situation in which the spacecraft attitude is maintained in a passive way (McInnes, 1998b, 2003; Quarta and Mengali, 2009, 2011a).

The paper is organized as follows. The spacecraft motion in a CR3BP is briefly discussed under the assumption that the propulsion system provides a radial thrust with respect to the more massive body. The corresponding equations of motion are derived in a dimensionless form to simplify the study of AEPs. The mathematical model of a generalized sail is then introduced and it is shown that the AEPs can be described in geometrical terms by varying the value of the sail's performance parameter. Three different families of equilibrium points, located on suitable loci, are shown to exist. Finally, the linear stability of the equilibrium points is discussed.

\section{Mathematical Model}

Consider the motion of a spacecraft under the gravitational effect of two celestial bodies $P_{1}$ and $P_{2}$, with masses $m_{1}$ and $m_{2}$ (assume that $m_{2} \leq m_{1}$ ). The two celestial bodies, which affect the motion of the spacecraft without being affected by the spacecraft themselves, cover a circular orbit about their center of mass $C$ with a constant angular velocity $\boldsymbol{\omega}$ whose modulus is

$$
\omega \triangleq\|\boldsymbol{\omega}\|=\sqrt{\frac{G\left(m_{1}+m_{2}\right)}{l^{3}}}
$$

where $l$ is the distance between the attractors and $G$ is the universal gravitational constant.

Let $\mathcal{T}(C ; x, y, z)$ be a synodic reference frame with origin at $C$ and unit vectors $\hat{\boldsymbol{i}}, \hat{\boldsymbol{j}}$, and $\hat{\boldsymbol{k}} \triangleq \boldsymbol{\omega} / \omega$. Referring to Fig. 1, the $(x, y)$ plane coincides with the orbital plane of the attractors, and the $x$ axis is in the direction from $P_{1}$ to $P_{2}$.

At a generic time instant the angular position of $x$ is defined by means of the true anomaly $\nu \geq 0$, which is measured anticlockwise from some reference direction belonging to the $(x, y)$ plane. Let $\boldsymbol{\rho}_{1}\left(\boldsymbol{\rho}_{2}\right)$ be the dimensionless vector from $P_{1}\left(P_{2}\right)$ to the spacecraft, and let $\mu \triangleq m_{2} /\left(m_{1}+m_{2}\right) \in(0,0.5]$ be the 
dimensionless mass of $P_{2}$, which univocally locates the position of the two attractors along the $x$ axis, see Fig. 1. Although in the Solar System the highest value of $\mu$ is about $10^{-3}$ (when $P_{1}$ is the Sun),

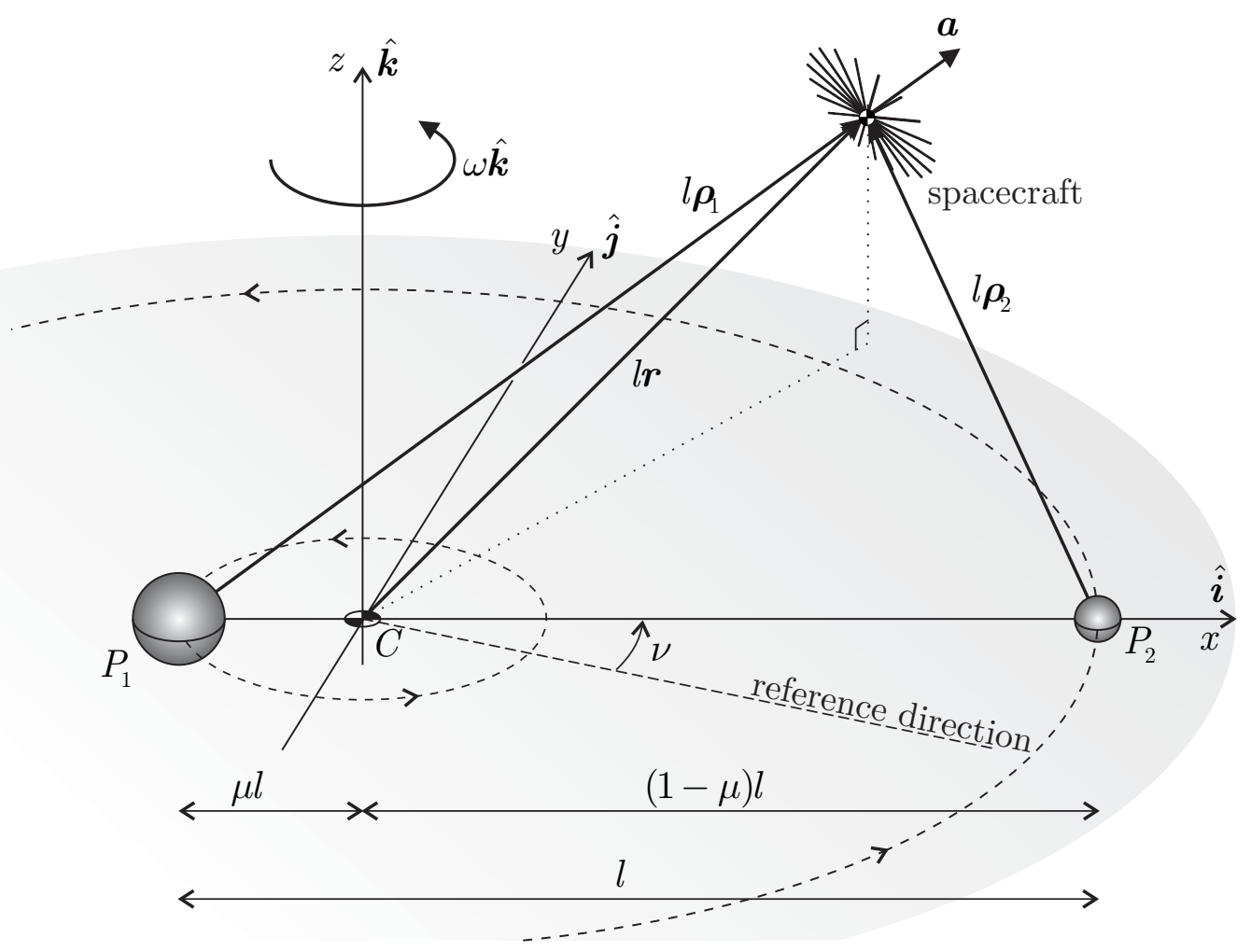

Fig. 1 Reference frame in the circular restricted three-body problem.

the range $(0,0.5]$ for the parameter $\mu$ is used, in this work, to achieve fully general results with the proposed mathematical model. Moreover, the selected range is useful to obtain a comparison with the classical results of the existing literature (Simmons et al., 1985) where the same variation range for the dimensionless mass $\mu$ is adopted.

Assume that the spacecraft propulsive acceleration $\boldsymbol{a}$ is parallel to $\boldsymbol{\rho}_{1}$ and is given by the following relationship

$$
\boldsymbol{a}=f \frac{G m_{1}}{l^{2}} \hat{\boldsymbol{\rho}}_{1}
$$

where $\hat{\boldsymbol{\rho}}_{1}=\boldsymbol{\rho}_{1} /\left\|\boldsymbol{\rho}_{1}\right\|$, and the magnitude of the dimensionless acceleration $f$ depends on the available propulsion system technology. 
According to Battin (1987), the spacecraft equations of motion in the synodic frame are:

$$
l \ddot{\boldsymbol{r}}+2 l \boldsymbol{\omega} \times \dot{\boldsymbol{r}}+l \boldsymbol{\omega} \times(\boldsymbol{\omega} \times \boldsymbol{r})+\frac{G m_{1}}{l^{2} \rho_{1}^{3}} \boldsymbol{\rho}_{1}+\frac{G m_{2}}{l^{2} \rho_{2}^{3}} \boldsymbol{\rho}_{2}=\boldsymbol{a}
$$

where $\boldsymbol{r}$ is the dimensionless vector that defines the spacecraft position with respect to $C, \rho_{1} \triangleq\left\|\boldsymbol{\rho}_{1}\right\|$, $\rho_{2} \triangleq\left\|\boldsymbol{\rho}_{2}\right\|$ and $\boldsymbol{a}$ is given by Eq. (2). Bearing in mind that $\hat{\boldsymbol{\omega}} \equiv \hat{\boldsymbol{k}}$, the equations of motion in dimensionless form can be obtained by using the true anomaly $\nu$ as the independent variable. The result is

$$
\boldsymbol{r}^{\prime \prime}+2 \hat{\boldsymbol{k}} \times \boldsymbol{r}^{\prime}+\hat{\boldsymbol{k}} \times(\hat{\boldsymbol{k}} \times \boldsymbol{r})+\frac{1-\mu}{\rho_{1}^{3}} \boldsymbol{\rho}_{1}+\frac{\mu}{\rho_{2}^{3}} \boldsymbol{\rho}_{2}=\frac{f(1-\mu)}{\rho_{1}} \boldsymbol{\rho}_{1}
$$

where the prime symbol denotes a derivative taken with respect to $\nu$. Note that, according to Fig. 1

$$
\boldsymbol{r}=\boldsymbol{\rho}_{1}-\mu \hat{\boldsymbol{i}} \quad, \quad \boldsymbol{\rho}_{2}=\boldsymbol{\rho}_{1}-\hat{\boldsymbol{i}}
$$

As a result, Eq. (4) can be rearranged and written as a function of the vector $\boldsymbol{\rho}_{1}$ only.

\subsection{Artificial Equilibrium Points}

The positions of AEPs are obtained by substituting Eqs. (5) into Eq. (4) and enforcing the conditions $\boldsymbol{r}^{\prime \prime}=0$ and $\boldsymbol{r}^{\prime}=0$. The result can be expressed in compact form as

$$
d_{k} \hat{\boldsymbol{k}}+d_{i} \hat{\boldsymbol{i}}=d_{\rho} \hat{\boldsymbol{\rho}}_{1}
$$

where

$$
d_{k} \triangleq \boldsymbol{\rho}_{1} \cdot \hat{\boldsymbol{k}} \quad, \quad d_{i} \triangleq \mu\left(1-\frac{1}{\rho_{2}^{3}}\right) \quad, \quad d_{\rho} \triangleq \rho_{1}-\frac{1-\mu}{\rho_{1}^{2}}-\frac{\mu \rho_{1}}{\rho_{2}^{3}}+f(1-\mu)
$$

An analysis of Eq. (6) provides the loci of AEPs and the corresponding value of the dimensionless acceleration $f$ required to maintain such equilibrium points. In what follows, the AEPs will be distinguished according to their different geometrical positions. 


\subsubsection{Triangular-type points}

The triangular-type points are obtained by looking for the values of $f, \rho_{2}$, and $\boldsymbol{\rho}_{1}$ in correspondence of which the coefficients $d_{k}, d_{i}$, and $d_{\rho}$ of Eq. (7) are all equal to zero, viz

$$
\boldsymbol{\rho}_{1} \cdot \hat{\boldsymbol{k}}=0 \quad, \quad \rho_{2}=1 \quad, \quad f=\rho_{1}\left(\frac{1}{\rho_{1}^{3}}-1\right)
$$

As a result, the locus of AEPs is constituted by a circle, with center $P_{2}$ and radius $l$, belonging to the $(x, y)$ plane (see the succeeding Fig. 4). For a given point on the locus, that is, for a given value of $\rho_{1} \in(0,2]$, the required value of $f$ can be calculated using the third equation of $(8)$. The name triangular type points comes from the fact that in the absence of any thrust $(f=0)$, Eq. (8) provides $\rho_{1} \equiv \rho_{2}=1$, which corresponds to the position of the classical Lagrange triangular points $\rho_{1_{L_{4}}} \equiv \rho_{2_{L_{4}}}=1$, and $\rho_{1_{L_{5}}} \equiv \rho_{2_{L_{5}}}=1$

\subsubsection{Collinear points}

Assume now that $d_{k}=0$, but $d_{i} \neq 0$ and $d_{\rho} \neq 0$. From Eq. (6) $d_{i} \hat{\boldsymbol{i}}=d_{\rho} \hat{\boldsymbol{\rho}}_{1}$, which implies that $\hat{\boldsymbol{\rho}}_{1}$ and $\hat{\boldsymbol{i}}$ are parallel. Accordingly, the AEPs belong to the $x$ axis and are such that

$$
d_{\rho}=d_{i} \hat{\boldsymbol{\rho}}_{1} \cdot \hat{\boldsymbol{i}}
$$

The second equation of $(5)$ states that $\rho_{2}=\left|\rho_{1} \hat{\boldsymbol{\rho}}_{1} \cdot \hat{\boldsymbol{i}}-1\right|$. The latter relationship can be substituted into Eq. (6) to find the dimensionless acceleration required to obtain the collinear AEPs. After some algebraic manipulations the result is:

$$
f=\frac{\mu}{(1-\mu) \hat{\boldsymbol{\rho}}_{1} \cdot \hat{\boldsymbol{i}}}\left(1+\frac{\rho_{1} \hat{\boldsymbol{\rho}}_{1} \cdot \hat{\boldsymbol{i}}-1}{\left|\rho_{1} \hat{\boldsymbol{\rho}}_{1} \cdot \hat{\boldsymbol{i}}-1\right|^{3}}\right)+\frac{1}{\rho_{1}^{2}}-\frac{\rho_{1}}{1-\mu}
$$

As in the previous case, the condition $f=0$ in Eq. (10) provides the position of the three classical collinear Lagrange points $\rho_{1_{L_{1}}}, \rho_{1_{L_{2}}}$, and $\rho_{1_{L_{3}}}$. Recall that $L_{1}$ is between the two attractors, $L_{2}$ is placed on the positive $x$ axis beyond $P_{2}$, and $L_{3}$ is on the left of $P_{1}$. 


\subsubsection{Displaced points}

If $d_{k}, d_{i}$, and $d_{\rho}$ are all different from zero, Eq. (6) can be satisfied only provided that the AEPs belong to the $(x, z)$ plane, that is, if $y=0$. Such points will be referred to as displaced points, in analogy to the usual nomenclature for non-Keplerian orbits (Gong et al., 2009; Xu and Xu, 2008; Mengali and Quarta, 2009b), which are obtained by using low-thrust propulsion systems. A general definition of non-Keplerian orbits is provided in McKay et al. (2011). To define the position of displaced points, take the dot product of both sides of Eq. (6) by $\hat{\boldsymbol{k}}$ and by $\hat{\boldsymbol{i}}$. The result is:

$$
\begin{aligned}
& \frac{\mu}{\rho_{2}^{3}}=\mu-\boldsymbol{\rho}_{1} \cdot \hat{\boldsymbol{i}} \\
& f=\frac{1}{\rho_{1}^{2}}+\frac{\rho_{1}\left(\mu-\boldsymbol{\rho}_{1} \cdot \hat{\boldsymbol{i}}\right)}{1-\mu}
\end{aligned}
$$

where

$$
\begin{aligned}
& \boldsymbol{\rho}_{1} \cdot \hat{\boldsymbol{i}}=x / l+\mu \\
& \rho_{2}=\sqrt{(x / l+\mu-1)^{2}+(z / l)^{2}}
\end{aligned}
$$

Then substitute Eqs. (13) and (14) into (11) to get

$$
z / l= \pm \sqrt{(-l \mu / x)^{2 / 3}-(x / l+\mu-1)^{2}}
$$

while Eq. (12) provides the corresponding value of $f$.

The locus of displaced AEPs is qualitatively drawn in Fig. 2. Note that the locus is symmetrical with respect to the $x$ axis and the points are such that $x \in(-\mu, 0)$ or, equivalently, $\rho_{2}>1$, see Eq. (15). As expected, Eqs. (11) and (12) show that displaced points cannot be obtained if $f=0$. Indeed, the right hand side of (12) is the sum of two strictly positive terms and, therefore, it cannot vanish.

\subsection{Power radial thrust}

So far the AEPs positions have been expressed as a function of the dimensionless acceleration $f$. Now the study is specialized to the situation in which $f$ varies with the distance $\rho_{1}$ from the massive attractor 


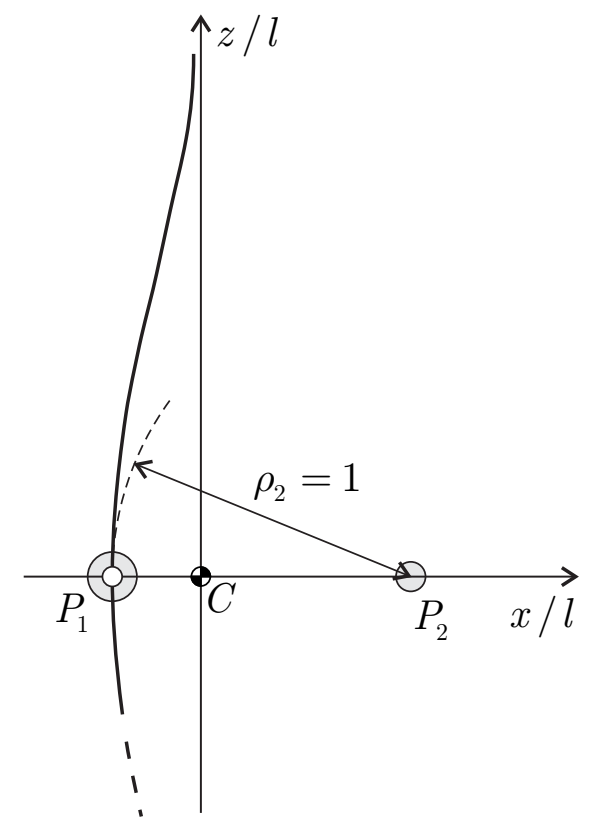

Fig. 2 Locus of displaced equilibrium points.

according to the following power law:

$$
f=\frac{\beta}{\rho_{1}^{\eta}}
$$

where $\eta$ is a real, nonnegative, coefficient that depends on the propulsion system type, while $\beta$ is a real parameter that quantifies the propulsion system performance. More precisely, for a given value of $\eta$, a decrease of $|\beta|$ implies a corresponding decrease in terms of propulsion system overall dimensions and mass (equivalently, for a given launch mass, the available payload mass increases).

Note that with the aid of Eq. (16) the equations of motion (3) can be written in compact form as

$$
l \ddot{\boldsymbol{r}}+2 l \boldsymbol{\omega} \times \dot{\boldsymbol{r}}=\nabla J^{\star}
$$

where $J^{\star} \triangleq J+\Psi$, and $J$ is the sum of the gravitational and centrifugal potential (Battin, 1987), while $\Psi$ represents the potential due to the propulsive acceleration, viz

$$
\Psi=\frac{\beta}{1-\eta} \frac{G m_{1}}{l^{2}} \frac{1}{\rho_{1}^{\eta-1}}
$$


Paralleling Battin (1987), it is possible to obtain a generalization of the Jacobi integral for a radial propulsive thrust as

$$
l^{2} \dot{\boldsymbol{r}} \cdot \dot{\boldsymbol{r}}-2 J^{\star}=\mathcal{C}
$$

where $\mathcal{C}$ is an integration constant.

A spacecraft equipped with a propulsion system capable of generating an acceleration in the form of Eq. (2), with $f$ given by Eq. (16), will be referred to as generalized sail. The reason is that if the massive body $m_{1}$ coincides with the Sun, Eq. (16) encompasses different types of propulsion systems, either currently available or under development (Racca, 2003; Frisbee, 2003). This is possible by simply specializing the value of $\eta$. For example $\eta=2$ describes the thrust provided by a solar sail (McInnes, 1999b) or a magnetic sail (Zubrin and Andrew, 1991), while $\eta \in[1,7 / 6]$ corresponds to an electric sail model (Janhunen and Sandroos, 2007; Mengali et al., 2008; Janhunen, 2010). Finally $\eta=0$ represents a constant propulsive acceleration, or a thrust that is independent of the distance from the massive attractor. Actually $\eta=0$ is consistent with a situation in which the propulsion system is either a minimagnetospheric plasma thruster (Winglee et al., 2000; Trask et al., 2004; Mengali and Quarta, 2006), or an electric thruster when the thrust is modulated such as to provide a constant thrust-to-mass ratio (Prussing and Coverstone, 1998; Mengali and Quarta, 2009a) and the power source is independent of the Sun-spacecraft distance, as in a nuclear-powered system. This happens, for example, when the power subsystem is represented by a radio-isotope thermoelectric generator (Hunt, 1993; Lyngvi et al., 2007). Note however that an electric thruster can also be characterized by a coefficient $\eta \neq 0$. In fact, the propulsive acceleration of a spacecraft of total mass $m_{s}$, equipped with an electric thruster of constant specific impulse $I_{s p}$, can be written as (Kechichian, 1995; Vadali et al., 2000; Mengali and Quarta, 2005)

$$
\boldsymbol{a}=\frac{2 \tau \sigma P}{m_{s} g_{0} I_{s p}} \hat{\boldsymbol{t}}
$$

where $\sigma$ is the constant thruster efficiency, $P$ is the engine maximum input power, $\tau \in[0,1]$ is the dimensionless throttle parameter $(\tau=1$ corresponding to the maximum thrust level for a given value of $P), g_{0}$ is the Earth's standard gravitational acceleration, and $\hat{\boldsymbol{t}}$ is the thrust unit vector.

In a solar-powered spacecraft, in which the electric power is supplied by solar arrays, the maximum input 
power $P$ is a function of the distance from the Sun (Sauer, 1978; Rayman and Williams, 2002), but also depends on the flight time due to the solar cells degradation (Richardson and Warren, 1971; Bourke, 1972; Saleh et al., 2002). If $P_{1}$ coincides with the Sun and the time degradation of solar cells is neglected, the maximum input power $P$ can be described through a rational function of $\rho_{1}$, that is, to a first order approximation (Sauer, 1978; Williams and Coverstone-Carroll, 1997)

$$
P=\frac{P_{r}}{\rho_{1}^{\eta}}
$$

where $P_{r}$ is a reference value that usually coincides with the maximum power generated at 1 Astronomical Unit from the Sun, and $\eta$ depends on the numerical coefficients that model the solar cells performance variation with the Sun-spacecraft distance. For example, using the numerical coefficients of Sauer (1978), the value of $\eta$ is about 1.6. Note that in an ideal case, that is, when the dependence of $P$ from $\rho_{1}$ is due only to the solar intensity radial variation, the exponent in Eq. (21) becomes $\eta=2$, and an inverse square law of $P$ with the Sun-spacecraft distance is obtained. In general, a reasonable assumption is that the value of $\eta$ varies within a range depending on the solar array characteristics. Finally note that the case of nuclear-powered spacecraft $(\eta=0)$ is recovered from Eq. (21) by observing that, for such a power source, $P \simeq P_{r}$.

Also note that for an electric propulsion system the instantaneous spacecraft mass is a function of time t. In fact, under the assumption that the thruster cannot be switched off and introducing the propellant mass flow rate $\dot{m}_{p}>0$, the instantaneous spacecraft mass is

$$
m_{s}=m_{s_{0}}\left(1-\frac{\dot{m}_{p}}{m_{s_{0}}} t\right)
$$

where $m_{s_{0}}$ is the initial mass. Substituting Eqs. (21) and (22) into Eq. (20) yields:

$$
\boldsymbol{a}=\frac{2 \sigma P_{r}}{m_{s_{0}} g_{0} I_{s p}}\left(\frac{\tau}{1-\dot{m}_{p} t / m_{s_{0}}}\right) \frac{\hat{\boldsymbol{t}}}{\rho_{1}^{\eta}}
$$

If the thrust level $\tau$ is modulated in such a way to counterbalance the mass loss, viz.

$$
\tau=1-\frac{\dot{m}_{p}}{m_{s_{0}}} t
$$


and the thrust is oriented parallel to $\hat{\boldsymbol{\rho}}_{1}$ (that is $\hat{\boldsymbol{t}} \cdot \hat{\boldsymbol{\rho}}_{1}= \pm 1$ ), Eq. (23) becomes:

$$
\boldsymbol{a}= \pm \frac{2 \sigma P_{r}}{m_{s_{0}} g_{0} I_{s p} \rho_{1}^{\eta}} \hat{\boldsymbol{\rho}}_{1}
$$

which is in the form of Eq. (2) provided that

$$
f= \pm \frac{2 \sigma P_{r} l^{2}}{G m_{1} m_{s_{0}} g_{0} I_{s p} \rho_{1}^{\eta}}
$$

Bearing in mind Eq. (16), the performance parameter $\beta$ of a solar-powered spacecraft is related to the main vehicle's characteristics through the equation:

$$
\beta= \pm \frac{2 \sigma P_{r} l^{2}}{G m_{1} m_{s_{0}} g_{0} I_{s p}}
$$

Note that, unlike a solar sail or an electric sail, an electric thruster can orient the thrust in any space direction. In particular, the propulsive acceleration can be oriented toward the attractor $P_{1}$ (that is, $\left.\hat{\boldsymbol{t}} \cdot \hat{\boldsymbol{\rho}}_{1}=-1\right)$ to obtain a negative value of $\beta$.

However, the use of an electric thruster as a primary propulsion system introduces a constraint on the total mission duration, as an AEP can be maintained only for a limited time interval. In fact, if $m_{p}<m_{s_{0}}$ is the onboard propellant mass, the mission duration $t_{m}$ is

$$
t_{m}=\frac{m_{p}}{\dot{m}_{p}}
$$

For example, assuming a propellant flow rate $\dot{m}_{p}=2.3 \mathrm{mg} / \mathrm{s}$, corresponding to the NASA's NSTAR ion thruster (Brophy, 2003, 2001), and a propellant mass $m_{p}=100 \mathrm{~kg}$, Eq. (28) states that an AEP can be maintained for about 500 days. Note however that the value of $\dot{m}_{p}$ (and the mission duration $t_{m}$ ) actually depends on the thruster setting, and a constant value of the propellant flow rate should be thought of as a first order approximation only. An in-depth discussion of the complex interactions between the thruster mathematical model and the mission performances is beyond the scope of this work, and the interested reader is referred to Quarta and Mengali (2011b). 
As previously stated, while $\eta$ defines the propulsion system type, $\beta$ quantifies the propulsion system performance. With reference to both solar sails and electric sails, $\beta$ can be expressed as a function of the maximum propulsive acceleration $a_{c}$ (referred to as characteristic acceleration) at a Sun-spacecraft distance equal to 1 Astronomical Unit. This is possible by combining Eqs. (2) and (16).

A brief discussion about the order of magnitude of the characteristic acceleration is now given. The only solar sails that have been successfully deployed in space so far, that is the JAXA's "IKAROS" (Higuchi and Ishimura, 2009) and the NASA's "NanoSail-D" (Johnson et al., 2011), have a characteristic acceleration of about 1-2 micro- $g_{0}$ ( $\beta$ is on the order of $10^{-3}$ ). However such a value is well below the current technological capabilities. For example proposals for NASA's Millennium Space Technology 9 mission include solar sails that produce thrust on the order of $0.58 \mathrm{~mm} / \mathrm{s}^{2}$ to values as high as $1.70 \mathrm{~mm} / \mathrm{s}^{2}$ (Lichodzeijewski and Derbes, 2006). Moreover it is widely accepted (Macdonald et al., 2006; Ozimek et al., 2009) that for a near term solar sail technology a reasonable performance is $a_{c}=0.5-1 \mathrm{~mm} / \mathrm{s}^{2}(\beta$ is on the order of $0.05-0.2$ ), especially when non-Keplerian orbits and AEPs are considered (West, 2008). A second generation of solar sails (Leipold et al., 1999) will probably have a higher characteristic acceleration. As a matter of fact, many studies exist with medium-high performance solar sails $\left(a_{c}=1-3 \mathrm{~mm} / \mathrm{s}^{2}\right.$, or $\beta$ around $0.2-0.5)$ and very-high performance solar sails $\left(a_{c}=3-6 \mathrm{~mm} / \mathrm{s}^{2}\right.$ or $\beta$ on the order of $\left.0.5-1\right)$ either for Solar System escape missions or for the fulfilment of highly-Non-Keplerian orbits (Sauer, 1999; Forward, 1991; McInnes, 1998a; Vulpetti and Scaglione, 1999; Vulpetti, 1997; Dandouras et al., 2004; Mengali and Quarta, 2007; Macdonald et al., 2010).

As far as the electric sail is concerned, the latest simulations by Janhunen (Janhunen, 2009, 2011) show that the propulsive thrust of a spacecraft with an in-flight mass of $100 \mathrm{~kg}$ is about $1 \mathrm{~N}$. Using these data, and according to the propulsion system's mathematical model discussed by Quarta and Mengali (2010), an electric sail is potentially capable of providing a characteristic acceleration of $2 \mathrm{~mm} / \mathrm{s}^{2}$ to a spacecraft with a total mass of $625 \mathrm{~kg}$ and a payload mass of about $215 \mathrm{~kg}$. In any case, it is important to recall that the value of $a_{c}$ is closely related to the value of the payload mass fraction for a given in-flight total mass. Therefore, the effective value of $\beta$ can be calculated only after that the payload mass has been selected. 
As a final remark, the succeeding discussion will be given in fully general terms, including values of $\eta$ and $\beta$ that do not correspond to any currently available propulsion system (this happens, for example, for $\eta>2$ ). The reason is to get an effective method for easily comparing the performance of different propulsion devices in generating AEPs. In this context the use of $\eta$ and $\beta$ as design parameters provides a certain degree of flexibility in dealing with exotic/future thrusters. For example it is worth mentioning that until the definition of the electric sail concept (that is, until Janhunen (2004)), the only realistic value for a propellantless propulsion system was $\eta=2$.

In what follows, for a given propulsion system type (that is, for a given value of $\eta$ ) the problem is to find the value of $\beta$ required for an AEP to be placed at a prescribed distance $\rho_{1}$ from the massive attractor. The pairs $\left(\beta, \rho_{1}\right)$ describe a locus, in the synodic reference frame, whose shape can be obtained for each of the three different families of AEPs. This matter is discussed in the next sections.

\subsubsection{Triangular-type points}

For triangular-type equilibrium points substitute Eq. (16) into Eq. (8) to get an expression of $\beta$ as a function of the distance $\rho_{1} \in(0,2]$ and of the coefficient $\eta$. The result is

$$
\beta=\rho_{1}^{\eta+1}\left(\frac{1}{\rho_{1}^{3}}-1\right)
$$

whose shape is qualitatively shown in Fig. 3. For a fixed value of $\eta>2$ the contour line $\beta=\beta\left(\rho_{1}, \eta\right)$ presents a maximum with coordinates $\left(\rho_{1_{\beta_{\max }}}, \beta_{\max }\right)$, where

$$
\rho_{1_{\beta_{\max }}}=\sqrt[3]{\frac{\eta-2}{\eta+1}} \quad, \quad \beta_{\max }=3 \sqrt[3]{\frac{(\eta-2)^{\eta-2}}{(\eta+1)^{\eta+1}}}
$$

In particular, when $\eta=2$ and $\beta>0$, that is, when the propulsion system is constituted by either a solar or a magnetic sail, in accordance with Simmons et al. (1985), Eq. (30) states that $\beta$ takes its maximum value $(\beta=1)$ as $\rho_{1}$ tends to zero. Clearly such a condition is not physically acceptable because the corresponding AEP would coincide with the attractor $P_{1}$. In the following those unattainable equilibrium points will be marked in the figures with a white circle. 


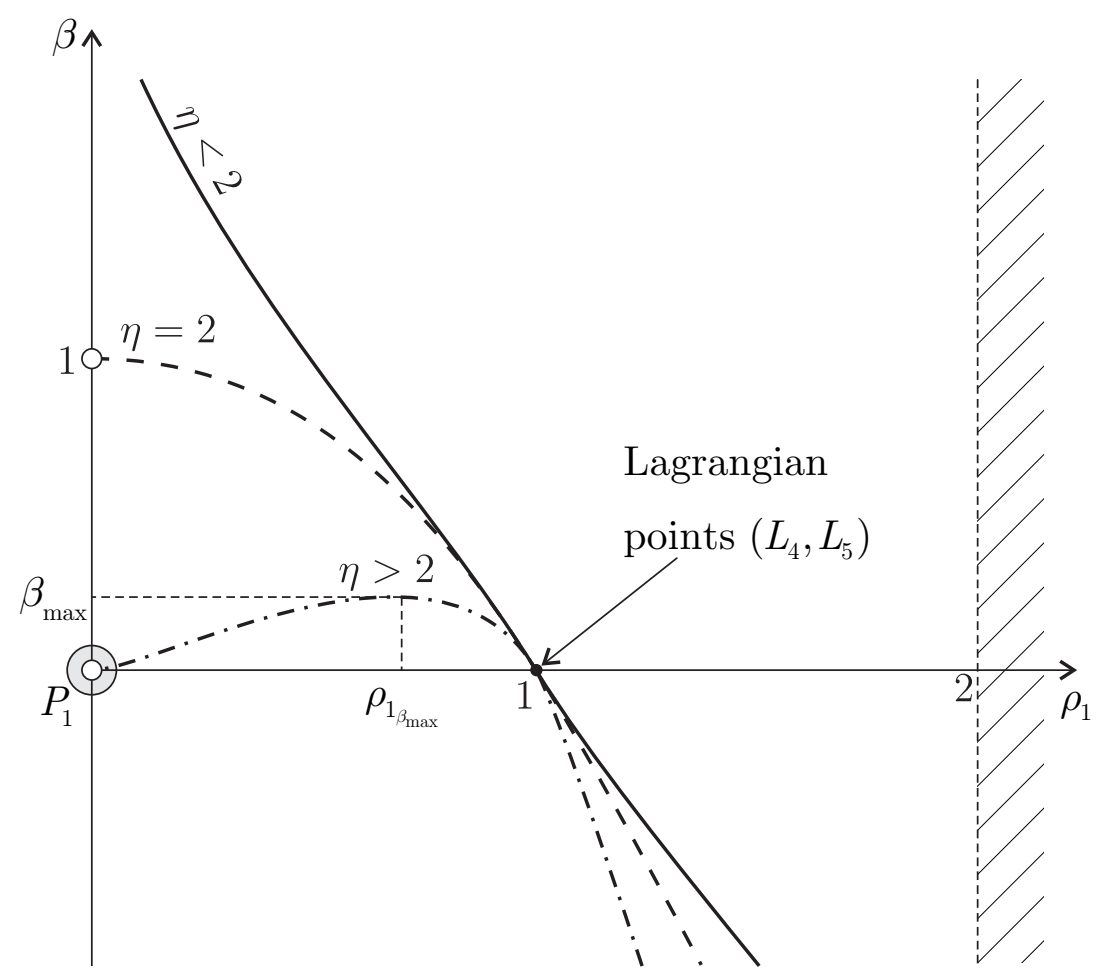

Fig. 3 Performance parameter $\beta$ as a function of $\rho_{1}$ and $\eta$ for triangular-type points.

The presence of a maximum value of the performance parameter $\beta$ implies that, for a given value of $\beta>0$, there exist either two AEPs (if $\eta \leq 2$ ) or four AEPs (if $\eta>2$ ). These points are at symmetrical positions with respect to the $x$ axis of the synodic reference frame, as is shown in Fig. 4. This figure illustrates the variation of $|\beta|$ on the locus of triangular-type points in the three cases $\eta<2, \eta=2$, and $\eta>2$. Note that for a fixed distance $\rho_{1}<1(>1)$, the parameter $\eta$ must be increased (decreased) to reduce the value of $|\beta|$ necessary to maintain such an AEP.

\subsubsection{Collinear points}

In this case the relationship between the performance parameter $\beta$ and the distance $\rho_{1}$ is obtained by substituting Eq. (16) into (10). To simplify the analysis of the function $\beta=\beta\left(\rho_{1}, \eta\right)$ it is useful to distinguish the collinear points according to their position with respect to the attractors, as shown in Fig. 5 . The variation of $\beta$ with $\rho_{1}$ for $L_{3}$-type points (the points in which $\hat{\boldsymbol{\rho}}_{1} \cdot \hat{\boldsymbol{i}}=-1$ ) is qualitatively shown in Fig. 6(a), while Fig. 6(b) illustrates the corresponding variation of the AEP position as a function of $\beta$. Similar to the previous triangular-type points, Fig. 6 (a) shows that the contour curves $\beta=\beta\left(\rho_{1}, \eta>2\right)$ take an absolute maximum whose value decreases as $\eta$ is increased. Once again, when $\eta=2$ the maximum 

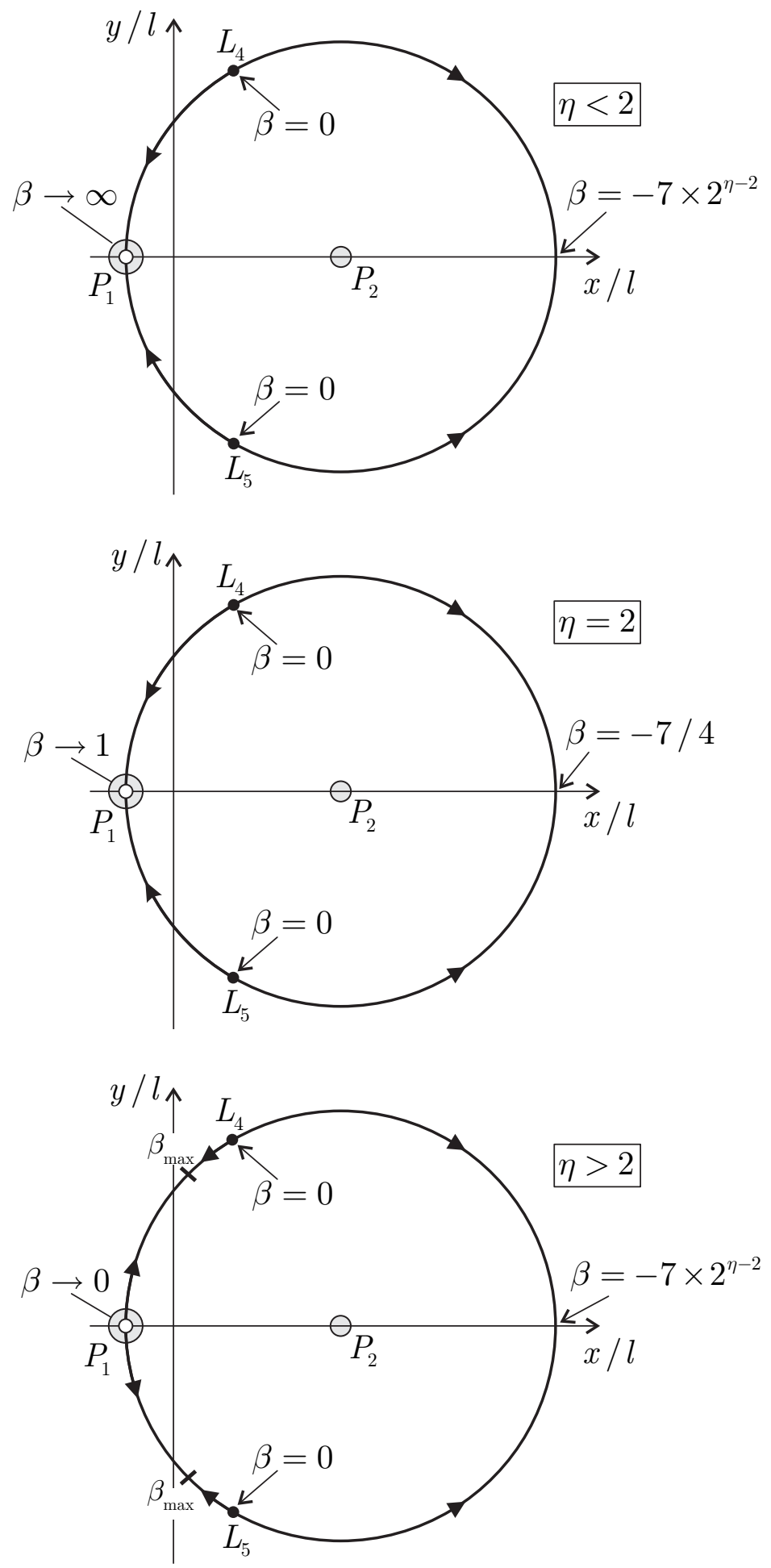

Fig. 4 Triangular-type equilibrium points locus (arrows indicate a $|\beta|$ increase). 


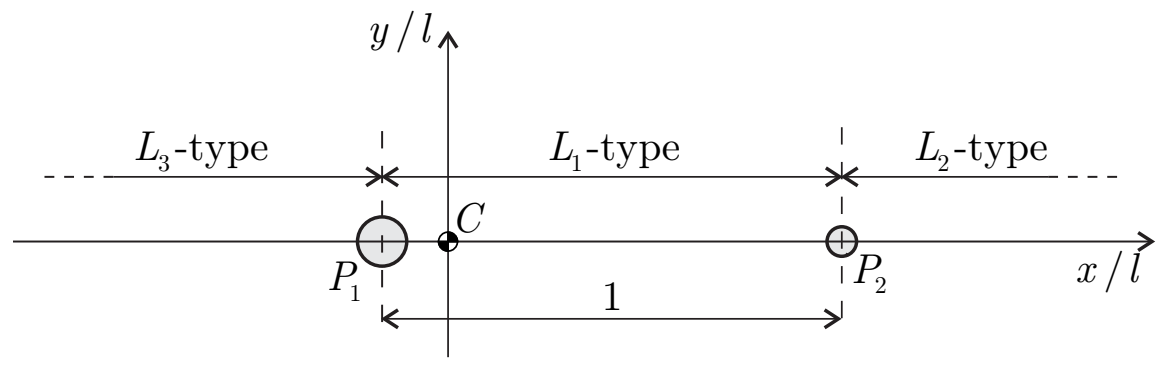

Fig. 5 Nomenclature of the collinear equilibrium points.

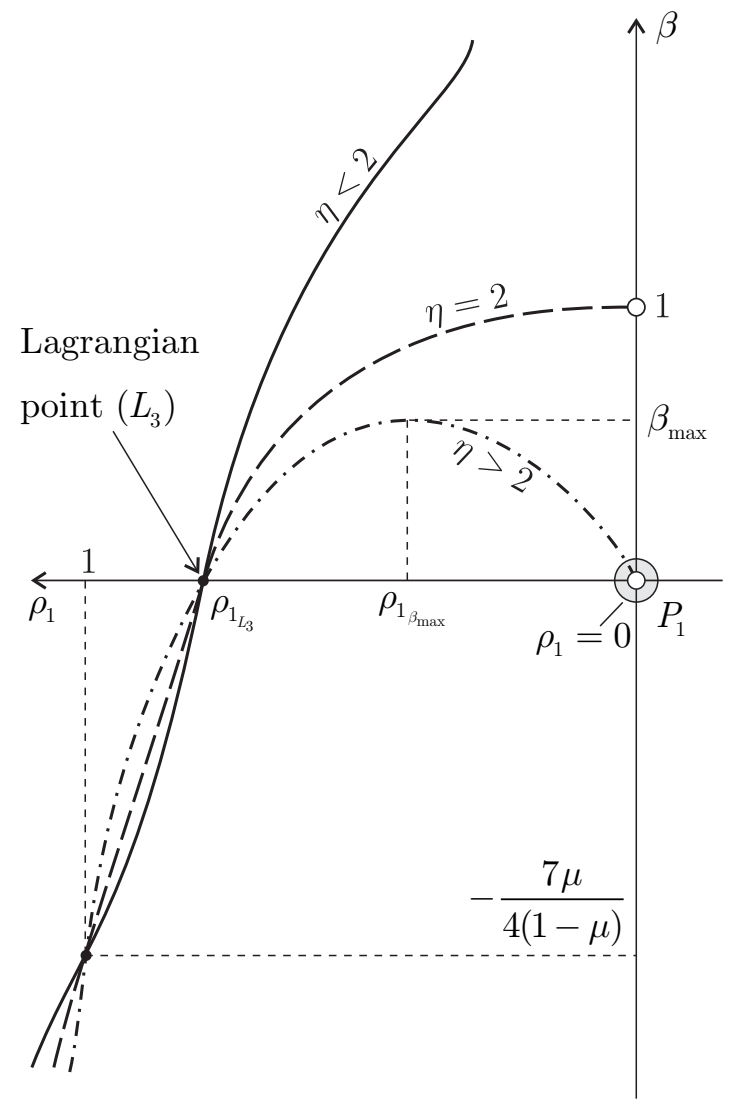

(a) Performance parameter $\beta$.

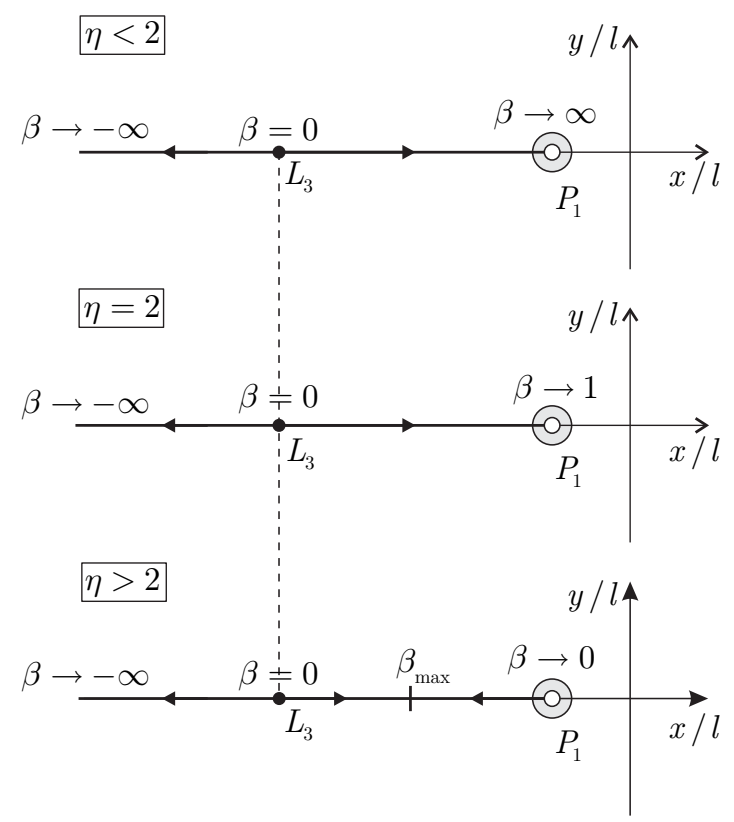

(b) Variation of $\beta$ on the locus.

Fig. 6 Performance parameter as a function of $\rho_{1}$ and $\eta$ for $L_{3}$-type equilibrium points locus (arrows indicate a $|\beta|$ increase).

is not attainable because it would coincide with the attractor $P_{1}$. If $\eta>2$ and $\beta \in\left(0, \beta_{\max }\right)$ two AEPs are obtained, whose position along the $x$ axis is between $P_{1}$ and the classical Lagrange point $L_{3}$. As the thrust tends to zero $(\beta=0)$, one AEP tends to coincide with $L_{3}$ and the other approaches $P_{1}$. Moreover, if $\beta$ is negative, that is, the propulsive acceleration is in the direction of the attractor $P_{1}$, there exists a single AEP on the left of $L_{3}$. Such a point can be theoretically placed at any distance from $P_{1}$ provided that a sufficient value of $\beta$ is available. Similar conclusions can be drawn for $L_{1}$-type points, that is, points in which $\hat{\boldsymbol{\rho}}_{1} \cdot \hat{\boldsymbol{i}}=+1$ and $\rho_{1}<1$, see Figs. $7(\mathrm{a})$ and $7(\mathrm{~b})$. In this case, $\eta$ being the same, a negative value of $\beta$ 
corresponds to an AEP between the Lagrangian point $L_{1}$ and the attractor $P_{2}$, while the condition $\beta>0$ permits to move the AEP toward the massive attractor $P_{1}$. Unlike the two previous cases an increase of $\eta$ corresponds now to a decrease of $|\beta|$.

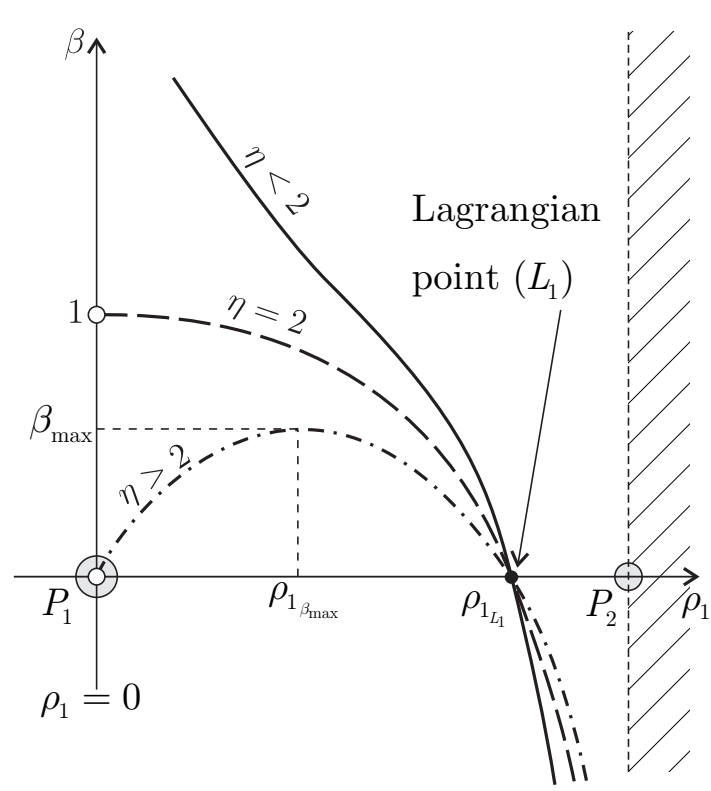

(a) Performance parameter $\beta$.

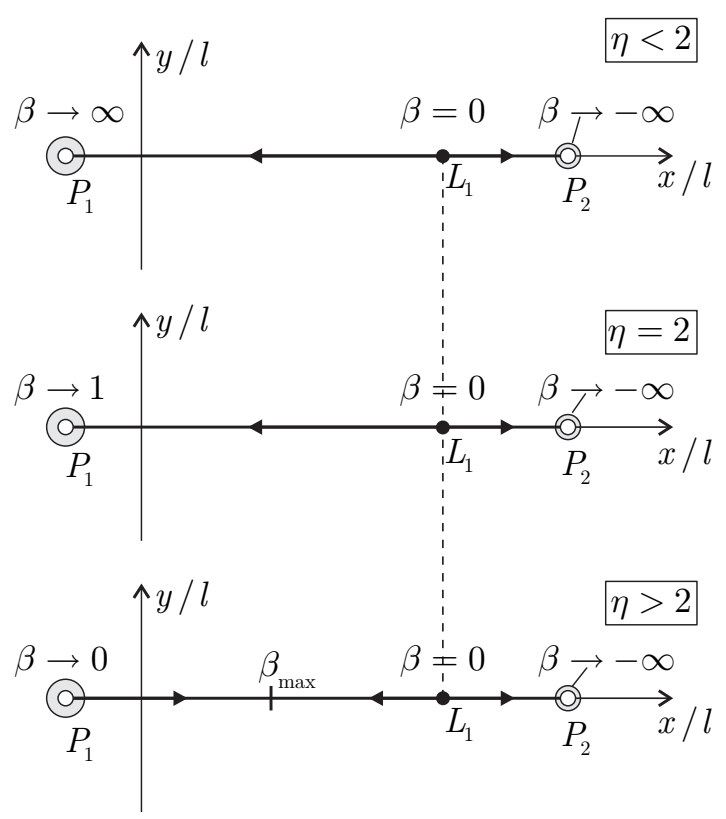

(b) Variation of $\beta$ on the locus.

Fig. 7 Performance parameter as a function of $\rho_{1}$ and $\eta$ for $L_{1}$-type equilibrium points locus (arrows indicate a $|\beta|$ increase).

Finally, the contour curves corresponding to the $L_{2}$-type points (when $\hat{\boldsymbol{\rho}}_{1} \cdot \hat{\boldsymbol{i}}=+1$ and $\rho_{1}>1$ ) are illustrated in Fig. 8(a). Fig. 8(a) shows that there exists a suitable value of $\eta$, referred to as $\widetilde{\eta}$, beyond which the contour curves $\beta=\beta\left(\rho_{1}, \eta>\widetilde{\eta}\right)$ display both a local minimum $\left(\beta_{\min }\right)$ and a maximum $\left(\beta_{\max }\right)$ at a distance $\rho_{1_{\beta_{\min }}}$ and $\rho_{1_{\beta_{\max }}}$ from the attractor $P_{1}$, respectively. The value of $\widetilde{\eta}$ is a function of the dimensionless mass of $P_{2}$ only and it decreases with $\mu$, as is shown in Fig. 9. Note however that the presence of the above points of maximum and minimum is confined to high value of $\eta$, that is, $\widetilde{\eta}>9.5$, see Fig. 9.

\subsubsection{Displaced points}

For displaced AEPs that belong to the plane $(x, z)$ of the synodic reference frame (except for the points on $x$ axis, see Fig. 2), the expression for $\beta$ is obtained by substituting Eq. (16) into (12):

$$
\beta=\rho_{1}^{\eta-2}\left(1+\frac{\mu}{1-\mu} \frac{\rho_{1}^{3}}{\rho_{2}^{3}}\right)
$$




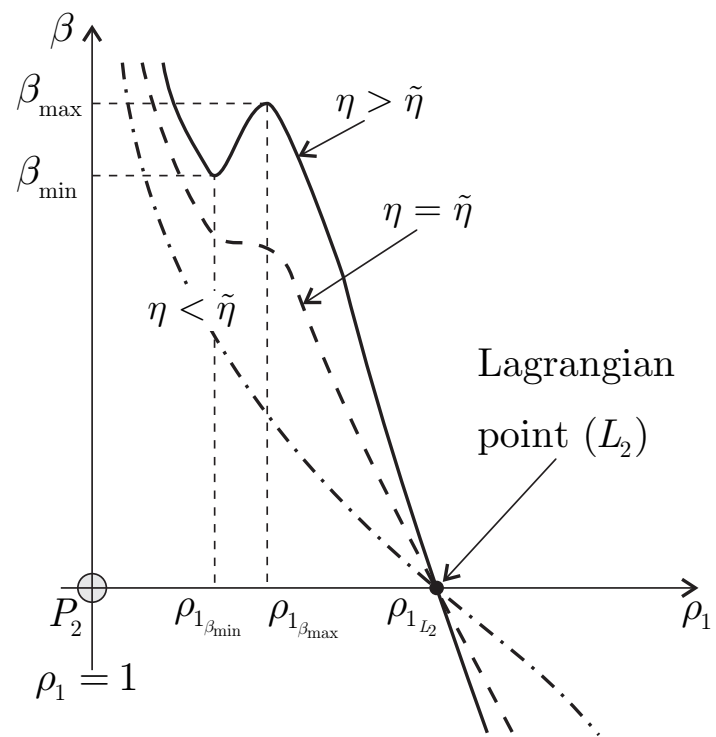

(a) Performance parameter $\beta$.

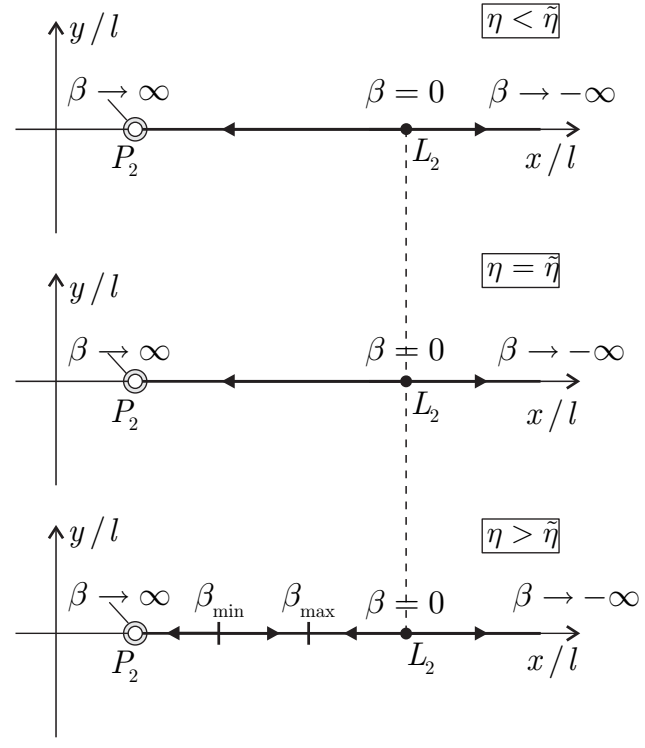

(b) Variation of $\beta$ on the locus.

Fig. 8 Performance parameter as a function of $\rho_{1}$ and $\eta$ for $L_{2}$-type equilibrium points locus (arrows indicate a $|\beta|$ increase).

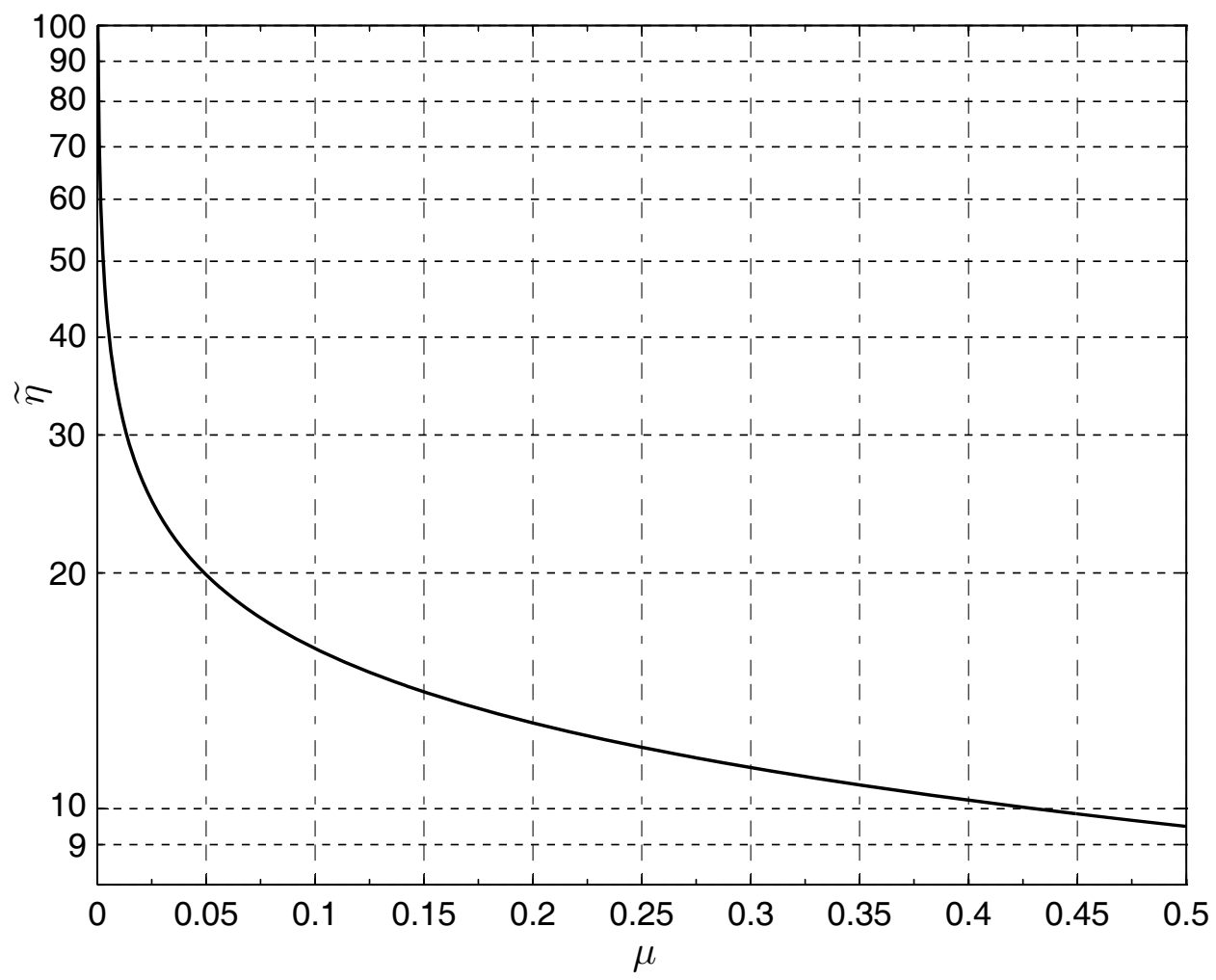

Fig. 9 Parameter $\widetilde{\eta}$ as a function of $\mu$ for $L_{2}$-type points.

where $\rho_{2}$ is given by Eq. (14). In particular for $\eta=2$ the result obtained with Eq. (31) is in agreement with Kunitsyn and Perezhogin (1978) and Simmons et al. (1985). Note that in the plane $(x, z)$ the distance $\rho_{1}$ 
is

$$
\rho_{1}=\sqrt{(x / l+\mu)^{2}+(z / l)^{2}}
$$

where $z / l$ is obtained from Eq. (15). Accordingly, $\beta$ is a function only of the dimensionless coordinate $x / l$. The function $\beta=\beta(x / l, \eta)$ is drawn in Fig. 10 .

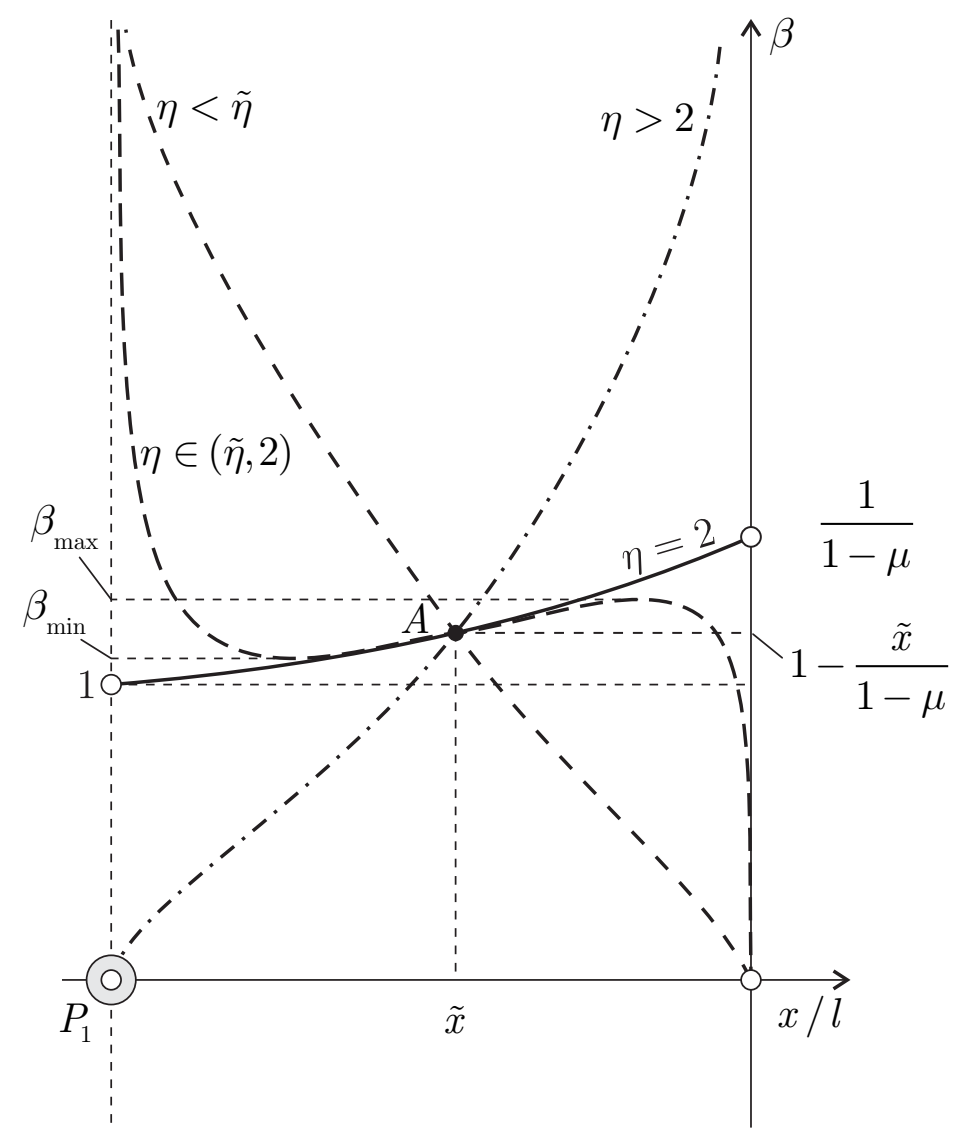

Fig. 10 Performance parameter $\beta$ as a function of $x / l$ and $\eta$ for displaced points.

As for the $L_{2}$-type points, it can be shown that a suitable value $\widetilde{\eta}<2$ exists, such that the function $\beta=\beta(x / l, \eta)$ presents two stationary points when $\eta \in(\widetilde{\eta}, 2)$. Figure 11 shows how $\tilde{\eta}$ varies with $\mu$.

When $\eta \leq \widetilde{\eta}$ or $\eta>2$, only two equilibrium points exist for a given value of $\beta$. On the other hand, when $\eta \in(\widetilde{\eta}, 2)$, there is a range of $\beta \in\left[\beta_{\min }, \beta_{\max }\right]$, such that four or six equilibrium points are possible. The different kinds of AEP locus are illustrated in Fig. 12, where the point $A$ corresponds to the condition $\rho_{1}=1$. Note that for a given $\rho_{1}<1(>1)$ it is necessary to increase (decrease) $\eta$ to decrease the performance parameter $\beta$. In particular, according to Kunitsyn and Perezhogin (1978), and Simmons et al. (1985), if $\eta=2$ two equilibrium points exist only for $\beta$ ranging in the interval $(1,1 /(1-\mu))$. 


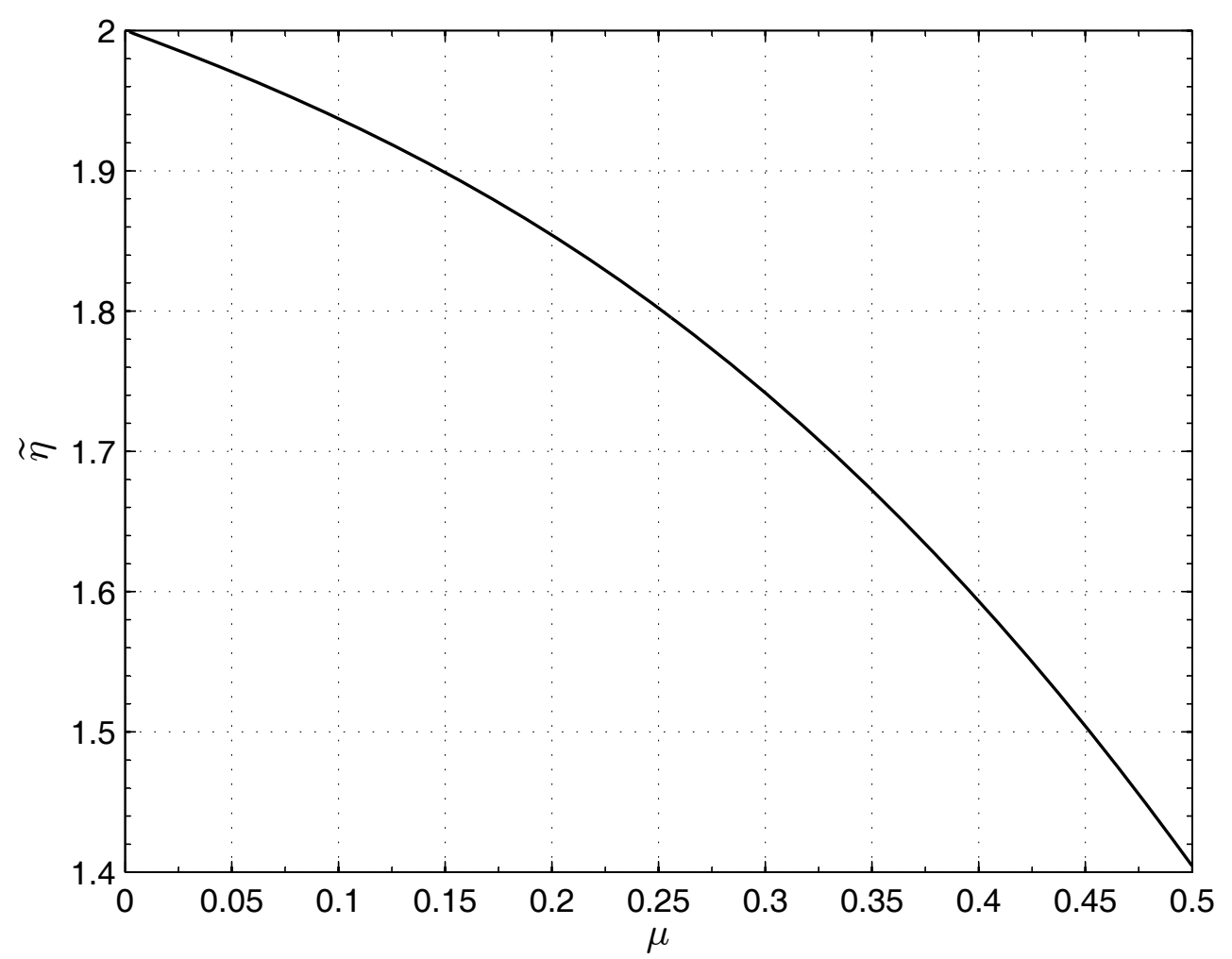

Fig. 11 Parameter $\widetilde{\eta}$ as a function of $\mu$ for displaced points.

\section{Linear Stability Analysis}

It is now possible to better characterize the spacecraft dynamical behavior by investigating its linear stability around the AEPs. Note that a linear analysis provides necessary conditions for stability and sufficient conditions for instability. To begin, introduce the second-order tensors $\boldsymbol{K}$ and $\boldsymbol{E}$ :

$$
\begin{aligned}
& \left.\boldsymbol{K} \triangleq \nabla\left[-\frac{1-\mu}{\rho_{1}^{3}} \boldsymbol{\rho}_{1}-\frac{\mu}{\rho_{2}^{3}} \boldsymbol{\rho}_{2}+\beta \frac{1-\mu}{\rho_{1}^{\eta+1}} \boldsymbol{\rho}_{1}-\hat{\boldsymbol{k}} \times(\hat{\boldsymbol{k}} \times \boldsymbol{r})\right]\right|_{\boldsymbol{r}_{0}} \\
& \hat{\boldsymbol{k}} \times \boldsymbol{r}=\boldsymbol{E} \cdot \boldsymbol{r}
\end{aligned}
$$

where $\boldsymbol{r}_{0}$ is the dimensionless vector that defines the position of a generic AEP with respect to the center of mass of the synodic frame. The variational equation of motion is obtained by linearizing the nonlinear equation (4) [where $f$ is given by Eq. (16)] around the equilibrium position $\boldsymbol{r}_{0}$. To this end, use the transformation $\boldsymbol{r}=\boldsymbol{r}_{0}+\delta \boldsymbol{r}$ and define a state vector $\boldsymbol{x} \triangleq\left[\delta \boldsymbol{r}, \delta \boldsymbol{r}^{\prime}\right]^{\mathrm{T}}$. The linearized equation takes the form

$$
\left[\boldsymbol{x}^{\prime}\right]_{\mathcal{T}}=\mathbb{M}[\boldsymbol{x}]_{\mathcal{T}}
$$



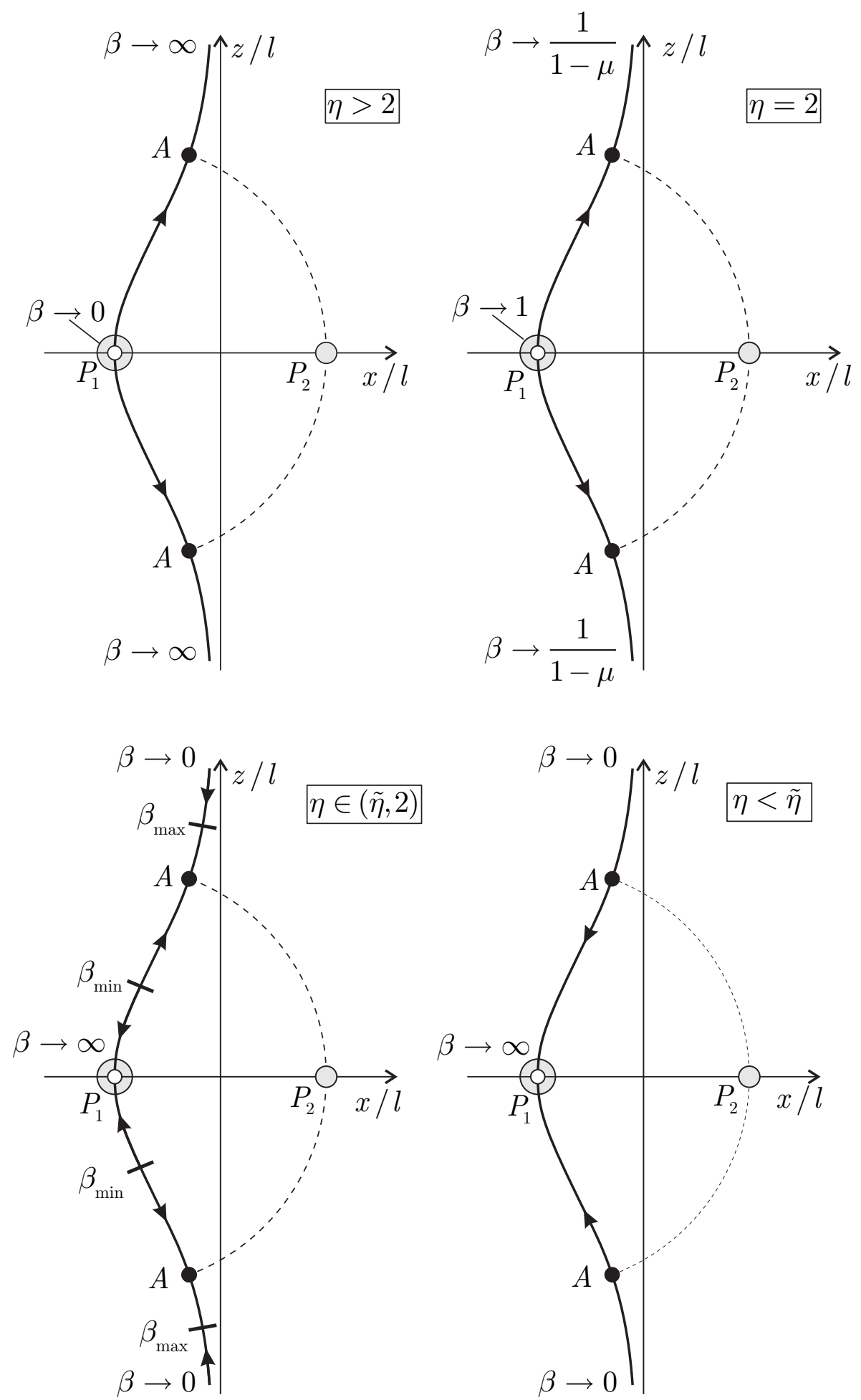

Fig. 12 Variation of $\beta$ on the displaced points locus (arrows indicate a $|\beta|$ increase).

with

$$
\mathbb{M}=\left[\begin{array}{cc}
\mathbb{O} & \mathbb{I} \\
\mathbb{K}^{\mathrm{T}} & -2 \mathbb{E}
\end{array}\right]
$$


where $\mathbb{O}$ is a $3 \times 3$ zero matrix, $\mathbb{I}$ is a $3 \times 3$ identity matrix, while matrices $\mathbb{K}$ and $\mathbb{E}$ contain the components of the second order tensors $\boldsymbol{K}$ and $\boldsymbol{E}$ in the synodic frame, that is

$$
\mathbb{K} \triangleq[\boldsymbol{K}]_{\mathcal{T}}=\left[\begin{array}{ccc}
k_{11} & k_{12} & k_{13} \\
k_{21} & k_{22} & k_{23} \\
k_{31} & k_{32} & k_{33}
\end{array}\right] \quad, \quad \mathbb{E} \triangleq[\boldsymbol{E}]_{\mathcal{T}}=\left[\begin{array}{ccc}
0 & -1 & 0 \\
1 & 0 & 0 \\
0 & 0 & 0
\end{array}\right]
$$

The value of all of matrix entries $k_{i j}$ (with $(i, j)=1,2,3$ ) is detailed in the appendix, as a function of $\rho_{1}$ (or $\rho_{2}$ ), $\eta$, and $\mu$, for the three different families of AEPs.

If $\lambda$ is the generic eigenvalue of $\mathbb{M}$, the characteristic equation of $\mathbb{M}$ can be shown to be in the form

$$
\lambda^{6}+a \lambda^{4}+b \lambda^{2}+c=0
$$

or

$$
s^{3}+a s^{2}+b s+c=0
$$

where $s \triangleq \lambda^{2}$ and $a, b$, and $c$ are real coefficients that depend on the entries $k_{i j}$. Note that if $\bar{\lambda}$ is a root of Eq. (38), then so is $-\bar{\lambda}$. For $\bar{\lambda}$ to be a stable eigenvalue, that is, a root with no positive real part, it is necessary that $\bar{\lambda}$ be both imaginary and a simple root of (38). Therefore, according to Abramowitz and Stegun (1965) and using the Descartes sign rule (Henrici, 1988), the system is marginally stable if and only if

$$
\Delta_{3}<0 \cap a \geq 0 \cap b \geq 0 \cap c>0
$$

where $\Delta_{3}$ is the discriminant of the cubic equation (39), that is:

$$
\Delta_{3} \triangleq \frac{1}{4}\left(c+\frac{2 a^{3}-9 a b}{27}\right)^{2}+\frac{1}{27}\left(b-\frac{a^{2}}{3}\right)^{3}
$$

As will be shown later, in some cases the characteristic equation can be factorized as

$$
(s-\widetilde{c})\left(s^{2}+\widetilde{a} s+\widetilde{b}\right)=0
$$


with suitable values of $\widetilde{a}, \widetilde{b}$, and $\widetilde{c}$, still depending on the entries $k_{i j}$. In such circumstances the necessary and sufficient conditions for the marginal stability become

$$
\left(\widetilde{a}^{2}-4 \widetilde{b}\right)>0 \cap \widetilde{a} \geq 0 \cap \widetilde{b}>0 \cap \widetilde{c}<0 \cap\left(\widetilde{c}^{2}+\widetilde{a} \widetilde{c}+\widetilde{b}\right) \neq 0
$$

Because both the conditions (40) and (43) affect only the coefficients of the characteristic equation, the stability of the linearized system can be investigated without the need of calculating the roots of Eq. (38). Moreover, with the aid of the results in the appendix, it can be verified that for a given system of attractors the coefficients of the characteristic equation are a function only of $\eta$ and $\rho_{1}$ (or $\rho_{2}$ ). Therefore, it is possible to draw the regions of stable AEPs for the different families of points, as is now discussed in detail.

\subsection{Collinear points stability}

The characteristic equation for collinear points is in the form of Eq. (42) with

$$
\widetilde{a}=4-k_{11}-k_{22} \quad, \quad \widetilde{b}=k_{11} k_{22} \quad, \quad \widetilde{c}=k_{33}
$$

where $k_{11}, k_{22}$, and $k_{33}$ are given in Eqs. (51)-(52) or (56)-(57). The stability conditions, provided by Eq. (43), must be specialized to the different families of AEPs.

For $L_{3}$-type points, taking into account that $k_{33}<0$ and $k_{22}<0$, the stability conditions (43) reduce to

$$
\left(4-k_{11}-k_{22}\right)^{2}-4 k_{11} k_{22}>0 \cap k_{11}<0
$$

where $k_{22}$ and $k_{11}$ are given by (52) and (54), respectively. First assume that $k_{11}<0$. The left inequality (45) can be written as

$$
\left(k_{11}-k_{22}\right)^{2}-8\left(k_{11}+k_{22}\right)+16>0
$$

which is always fulfilled because $k_{22}<0$. That means that $L_{3}$-type points are stable provided that $k_{11}<0$. From Eq. (54) the sign of $k_{11}$ is a function of $\partial \beta / \partial \rho_{1}$. According to Fig. 6(a), one concludes that $k_{11}<0$ 
if $\eta>2 \cap \rho_{1}<\rho_{1_{\beta_{\max }}}$, where $\rho_{1_{\beta_{\max }}}$ corresponds to the distance $\rho_{1}$ at which the function $\beta=\beta\left(\rho_{1}, \eta>2\right)$ attains its maximum value. The variation of $\rho_{1_{\beta_{\max }}}$ with $\eta$, for a given value of $\mu$, is qualitatively drawn in Fig. 13(a) along with the stability region of $L_{3}$-type points.

Similar considerations apply to $L_{1}$-type points, and the corresponding stability region is shown in gray in Fig. 13(b). Note that for both Figs. 13(a) and 13(b) the boundary of the stability region is to be excluded as it corresponds to the condition $k_{11}=0$. Figures $14(\mathrm{a})$ and $14(\mathrm{~b})$ show the stability regions corresponding to $\mu=0.01$. Finally, the stability conditions (43) for $L_{2}$-type points can be simplified by

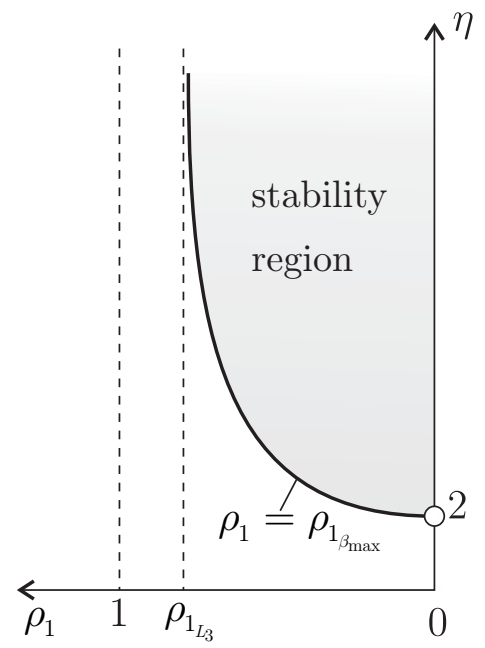

(a) $L_{3}$-type points.

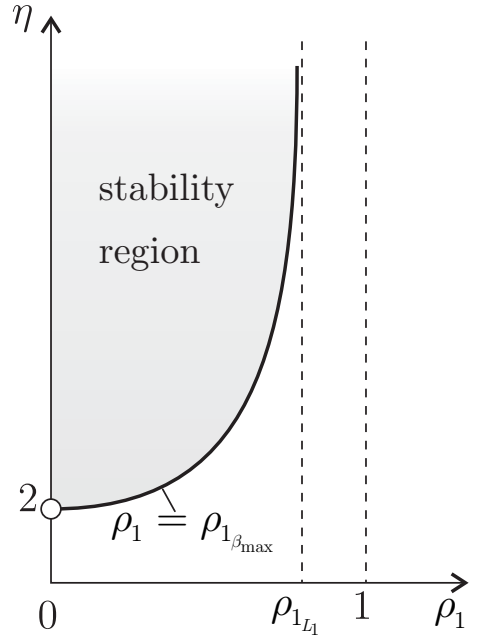

(b) $L_{1}$-type points

Fig. 13 Distance $\rho_{\beta_{\beta_{\max }}}$ as a function of $\eta$ (with $\mu$ given) for $L_{3}$ and $L_{1}$ type points.

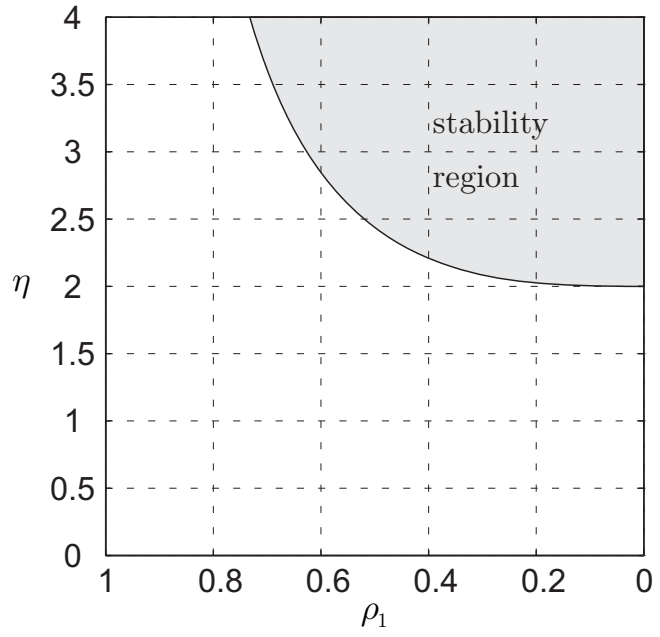

(a) $L_{3}$-type points.

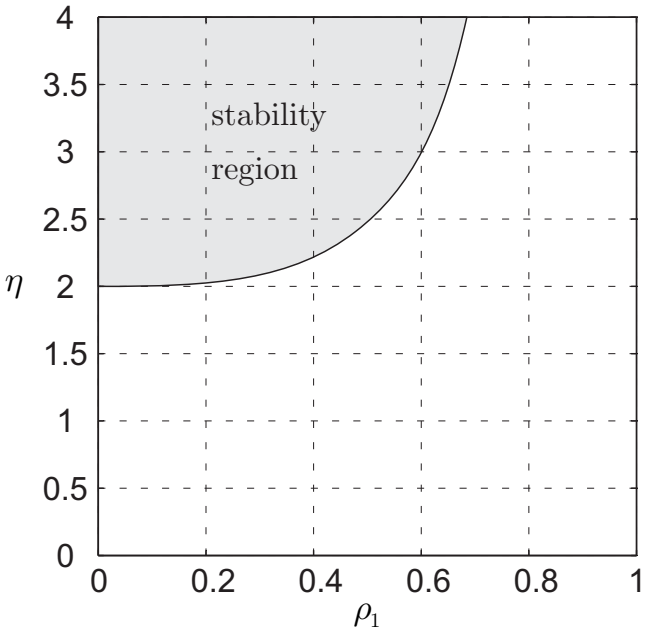

(b) $L_{1}$-type points.

Fig. 14 Stability region with $\mu=0.01$ for $L_{3}$ and $L_{1}$ type points. 
taking into account the actual value of $\rho_{1}$. In fact, when $\rho_{1}<2$ the stability conditions coincide with those shown in Eq. (45) in which $k_{11}$ and $k_{22}$ are now given by Eqs. (56) and (57). The study of the stability region proceeds as in case of $L_{3}$-type points, and the corresponding graphical results are summarized in Fig. 15. Note that the value $\widetilde{\eta}$ shown in Fig. 15 depends on $\mu$ and can be taken from Fig. 9.

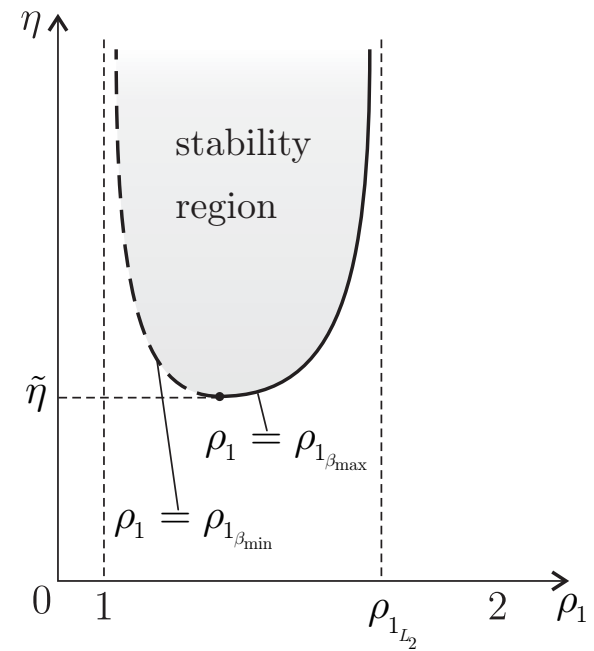

Fig. 15 Position of stationary values of $\beta$ for $L_{2}$-type points.

On the contrary, when $\rho_{1}>2$ the conditions (43) must be studied with a numerical approach. For a given value of $\mu$ the stability region (with the exclusion of the boundary line) can be drawn in the plane $\left(\rho_{1}, \eta\right)$. An example of this kind of study is shown in Fig. 16 where the region corresponding to $\rho_{1}<2$ is also displayed for the sake of completeness. Note that the equilibrium point corresponding to $\rho_{1}=2$ is unstable. In fact in such a case $\widetilde{b}=0$, see Eqs. (43).

Figure 16 shows that for $\eta=0$ there exist stable points only provided that $\rho_{1}>2$. Note that the results of Fig. 16 do not match that of Morimoto et al. (2007) because of the different assumptions made on the thrust direction. In fact Morimoto et al. (2007) uses a constant thrust vector, that is, a vector with a constant modulus and a fixed direction. Accordingly, in Morimoto et al. (2007) the gradient of the propulsive acceleration is equal to zero. In this paper, instead, when $\eta=0$ the thrust modulus is constant (see Eq. (16)), but its orientation is always radial, see Eq. (2). Therefore, when the system is perturbed, the gradient of the propulsive acceleration is different from zero, see Eq. (33).

The existence of the stability regions shown in Fig. 16 is confirmed by numerical simulations of the perturbed trajectory. For example, according to Fig. 16, the $L_{2}$-type collinear point located at $\rho_{1}=1.5$ 


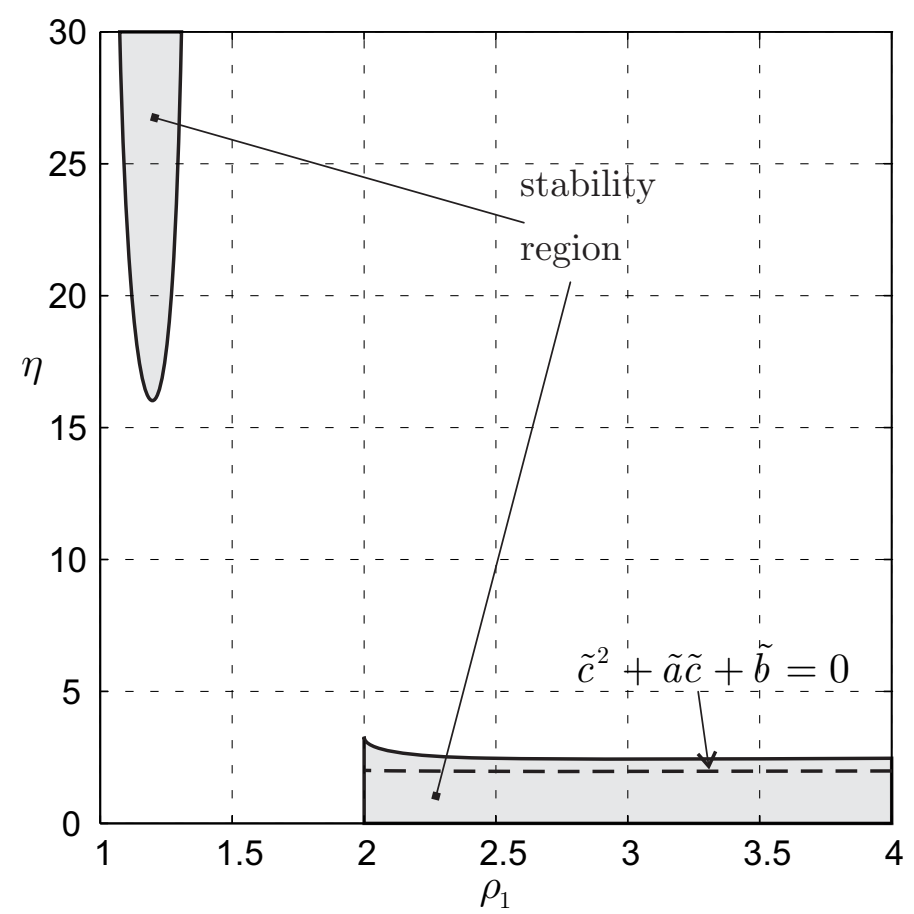

Fig. 16 Stability region for $L_{2}$-type collinear points with $\mu=0.1$ (the dashed line is excluded).

is unstable both for $\eta=0$ and $\eta=1$ (see Figs. 17(a) and 17(c)). On the contrary, assuming $\rho_{1}=2.5$, Figs. 17(b) and 17(d) prove that the perturbed motion around AEP is actually stable.

3.2 Triangular points stability

The characteristic equation for triangular type points is in the form of Eq. (42) with

$$
\widetilde{a}=4-k_{11}-k_{22} \quad, \quad \widetilde{b}=k_{11} k_{22}-k_{12}^{2} \quad, \quad \widetilde{c}=k_{33}
$$

where $k_{11}, k_{22}$, and $k_{33}$ are given by Eqs. (60)-(62).

The conditions (43) (with the exception of $\tilde{c}<0$, which is always satisfied) must be studied numerically to find the stability region in the plane $\left(\rho_{1}, \eta\right)$. An example is shown in Fig. 18 for some values of $\mu$, including the critical value (Szebehely, 1967) $\mu^{\star} \triangleq(1-\sqrt{23 / 27}) / 2 \simeq 3.85209 \times 10^{-2}$, which defines the stability of the classic solution with $\rho_{1}=1$.

As long as $\mu \geq \mu^{\star}$, the plane $\left(\rho_{1}, \eta\right)$ contains two distinct stability regions, see Fig. 18(a), that exclude the case $\rho_{1}=1$. As $\mu$ is decreased, the two regions get closer and eventually they touch when $\mu=\mu^{\star}$, 


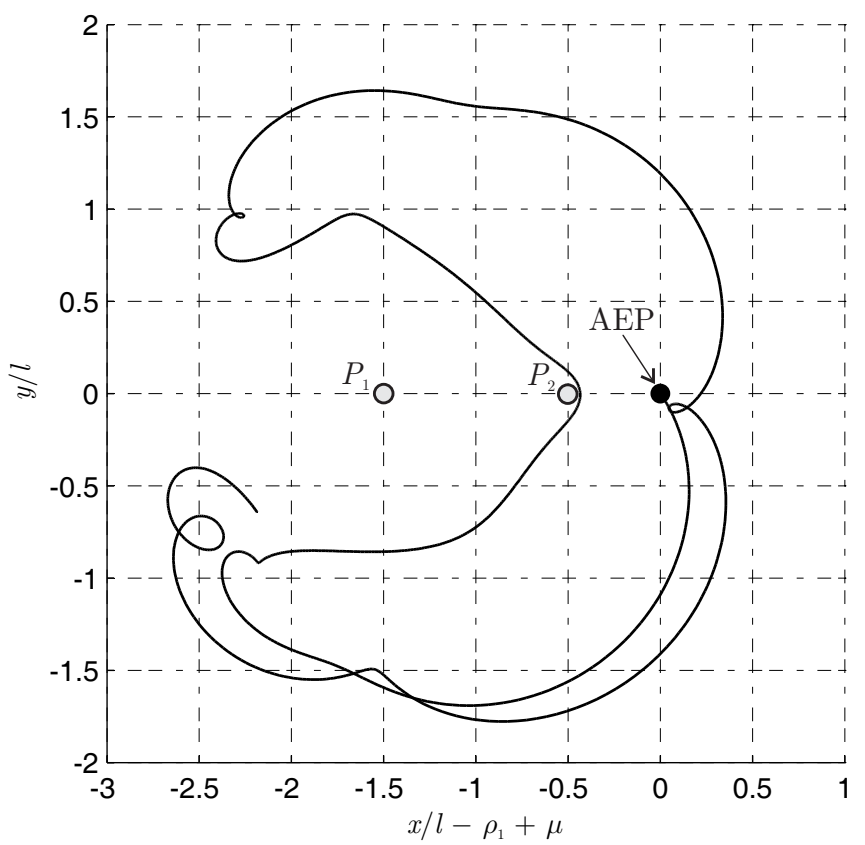

(a) $\rho_{1}=1.5$ and $\eta=0$ (unstable)

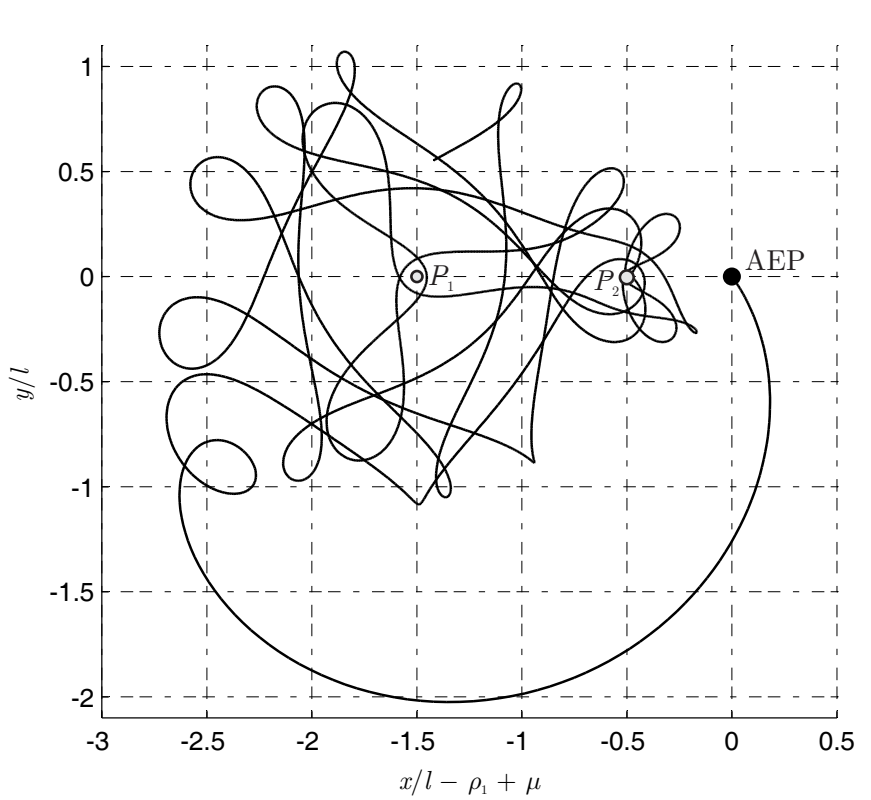

(c) $\rho_{1}=1.5$ and $\eta=1$ (unstable)

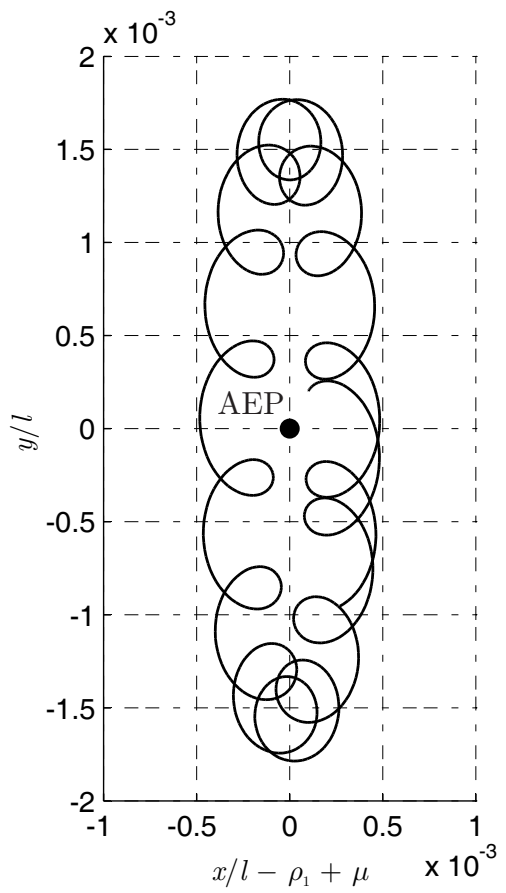

(b) $\rho_{1}=2.5$ and $\eta=0$ (stable)

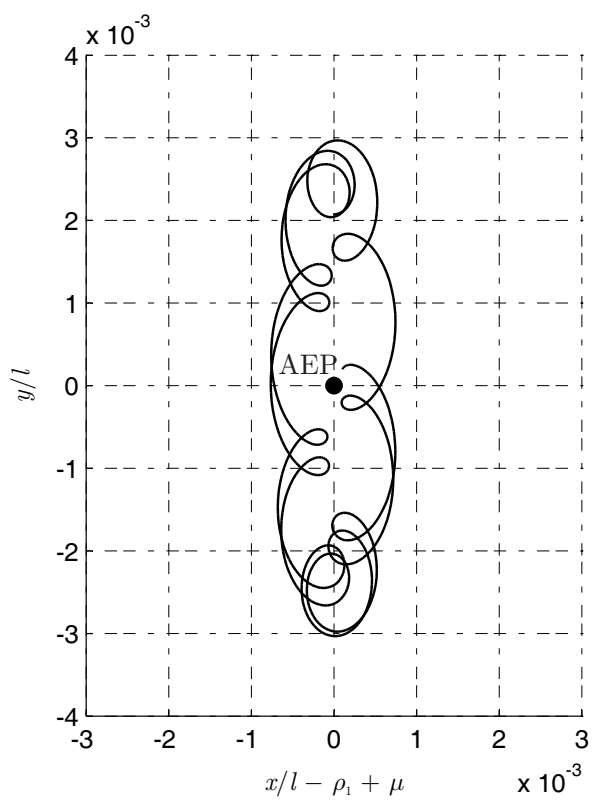

(d) $\rho_{1}=2.5$ and $\eta=1$ (stable)

Fig. 17 Numerically integrated perturbed trajectory (ten-periods time span) around an $L_{2}$-type collinear point with $\mu=0.1$.

see Fig. 18(b). Finally, if $\mu<\mu^{\star}$ there exists a single stability region in the plane $\left(\rho_{1}, \eta\right)$, as shown in Fig. 18(c). Note that, as in the previous case, the boundary line does not belong to the stability region. 


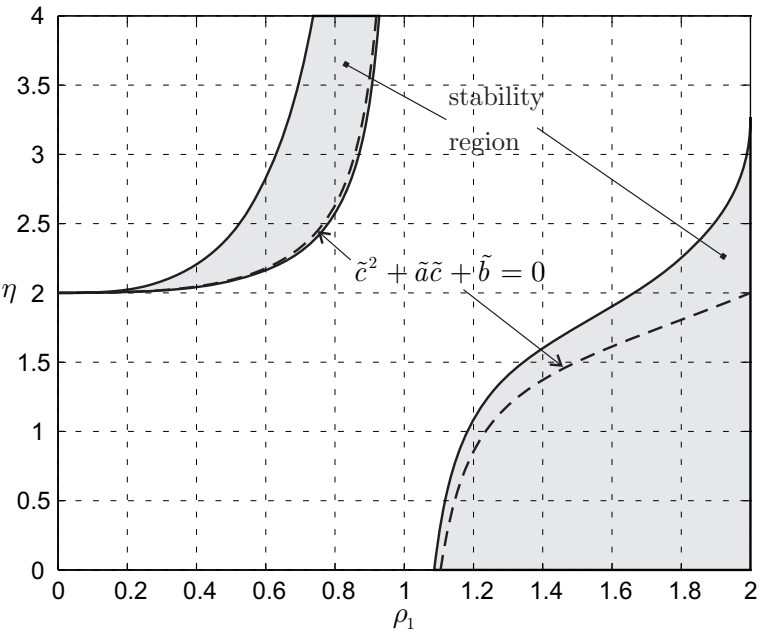

(a) $\mu=0.1$

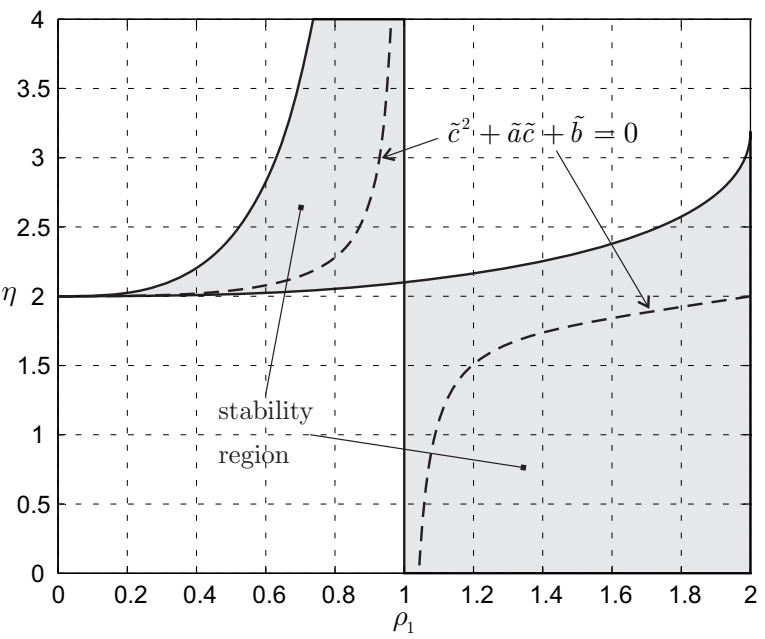

(b) $\mu=\mu^{\star} \simeq 3.85209 \times 10^{-2}$

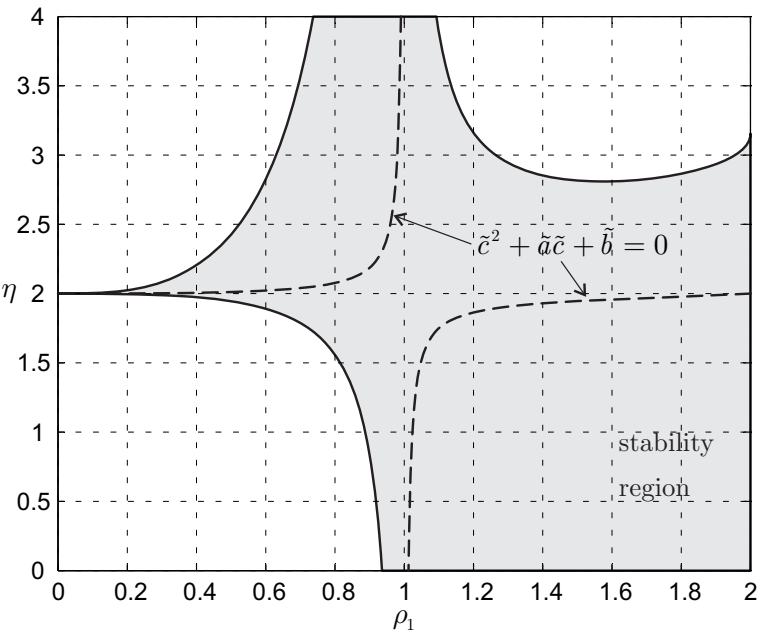

(c) $\mu=0.01$

Fig. 18 Stability region for triangular points for different values of $\mu$ (the dashed lines are excluded). 
3.3 Displaced points stability

The characteristic equation for displaced points is in the form of Eq. (39) in which, bearing in mind Eq. (68), the coefficients $a, b$, and $c$ can be written as

$$
a=3-k_{11}-k_{33} \quad, \quad c=k_{13}^{2}-k_{11} k_{33} \quad, \quad b=k_{11}-3 k_{33}-c
$$

where $k_{11}, k_{33}$, and $k_{13}$ are obtained from Eqs. (67), (69) and (70).

The stability region must be investigated with a numerical approach by looking for the pairs $\rho_{2}$ and $\eta$ that, for a given value of $\mu$, satisfy the condition (40). The results are summarized in Fig. 19, in which the stability region does not include the boundary line.

\section{Conclusions}

Under the assumption of a spacecraft subject to a radial propulsive acceleration with respect to the massive attractor, a new approach has been discussed for the analysis of artificial equilibrium points in the circular restricted three body problem. The concept of generalized sail is capable of describing, by means of a unified mathematical model, the position and the linear stability of AEPs for different propulsion systems of practical interest. Within this new model the spacecraft propulsive acceleration depends on the available propulsion system technology by means of two parameters, $\eta$ and $\beta$, one defining the propulsion system type, and the other that quantifies the propulsion system performance. While the paper contains an analysis about the order of magnitude of current and near future technological capabilities, the discussion is given in very general terms and, in particular, it includes values of $\eta$ and $\beta$ that do not correspond to any currently available propulsion system. The rationale is to provide a certain degree of flexibility in dealing with exotic/future thrusters and space missions. Likewise, although in the Solar System $\mu$ does not exceed about $10^{-3}$, the paper includes a discussion in which $\mu$ ranges in the interval $(0,0.5]$. The selected range is useful to obtain a comparison with classical results of the existing literature in which the same variation is assumed. The proposed model allows one to compare, either in an analytical or graphical form, the performance required by a given propulsion system to produce and maintain a prescribed AEP. Accordingly, it is possible to quickly obtain information about different propulsion systems and decide 


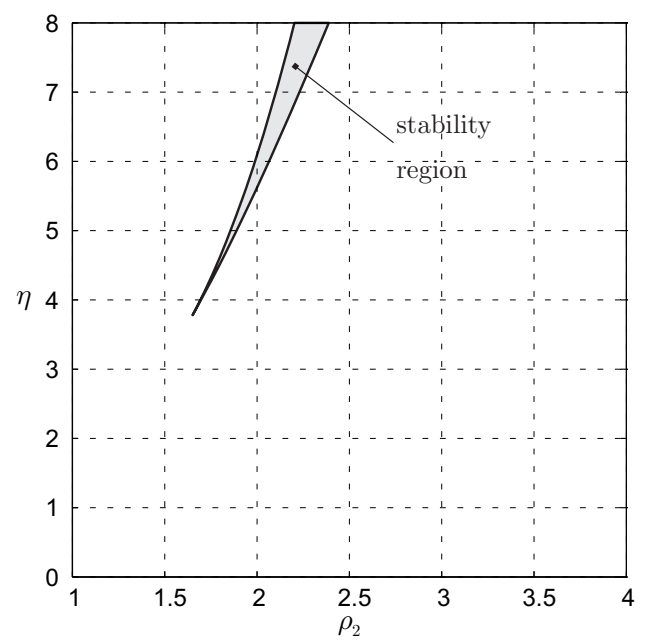

(a) $\mu=0.1$

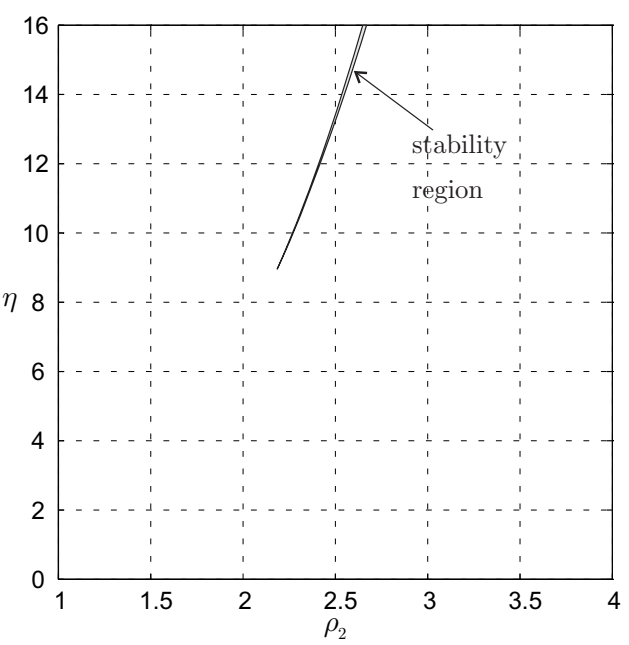

(b) $\mu=0.01$

Fig. 19 Stability region for displaced points for different values of $\mu$.

upon which one is better suited for a particular mission type. The proposed model can also be extended to deal with an elliptical restricted three body problem and with the more general situation in which the spacecraft thrust is not constrained to coincide with the radial direction. The latter aspects are currently under development. 


\section{A Entries of $\mathbb{K}$-matrix}

Consider the second-order tensor $\boldsymbol{K}$ defined by means of Eq. (33). The entries of the symmetric matrix $\mathbb{K}$, corresponding to the components of $\boldsymbol{K}$ in the synodic frame $\mathcal{T}(C ; x, y, z)$, are obtained observing that

$$
\mathbb{K}=\left[\frac{1-\mu}{\rho_{1}^{5}}\left(3\left[\boldsymbol{\rho}_{1}\right]_{\mathcal{T}}\left[\boldsymbol{\rho}_{1}\right]_{\mathcal{T}}^{\mathrm{T}}-\rho_{1}^{2} \mathbb{I}\right)+\frac{\mu}{\rho_{2}^{5}}\left(3\left[\boldsymbol{\rho}_{2}\right]_{\mathcal{T}}\left[\boldsymbol{\rho}_{2}\right]_{\mathcal{T}}^{\mathrm{T}}-\rho_{2}^{2} \mathbb{I}\right)-\mathbb{E}^{2}-\left.\beta \frac{1-\mu}{\rho_{1}^{\eta+3}}\left[(\eta+1)\left[\boldsymbol{\rho}_{1}\right]_{\mathcal{T}}\left[\boldsymbol{\rho}_{1}\right]_{\mathcal{T}}^{\mathrm{T}}-\rho_{1}^{2} \mathbb{I}\right]\right|_{\boldsymbol{r}_{0}}\right.
$$

where $\mathbb{E}$ is given by Eq. (37), and $\beta$ takes that particular value for which $\boldsymbol{r}_{0}$ corresponds to an equilibrium position. As a result, the entries of $\mathbb{K}$ take different expressions for the distinct families of AEPs.

A.1 Collinear points: $L_{3}$-type

The expression for $\beta$ is obtained by combining Eqs. (10) and (16), and

$$
\left[\boldsymbol{\rho}_{1}\right]_{\mathcal{T}}=-\left[\begin{array}{lll}
\rho_{1} & 0 & 0
\end{array}\right]^{\mathrm{T}} \quad, \quad\left[\boldsymbol{\rho}_{2}\right]_{\mathcal{T}}=-\left[\begin{array}{lll}
\left(\rho_{1}+1\right) & 0 & 0
\end{array}\right]^{\mathrm{T}}
$$

Therefore, Eq. (49) gives the following results

$$
\begin{aligned}
& k_{11}=2 \frac{1-\mu}{\rho_{1}^{3}}+2 \frac{\mu}{\left(\rho_{1}+1\right)^{3}}+1-\frac{\eta}{\rho_{1}}\left[\frac{1-\mu}{\rho_{1}^{2}}+\frac{\mu}{\left(\rho_{1}+1\right)^{2}}-\rho_{1}-\mu\right] \\
& k_{22}=k_{33}+1=\frac{\mu}{\rho_{1}}\left[\frac{1}{\left(\rho_{1}+1\right)^{3}}-1\right] \\
& k_{12}=k_{21}=k_{23}=k_{32}=k_{13}=k_{31}=0
\end{aligned}
$$

Note that Eq. (51) can also be rearranged into the following equivalent expression:

$$
k_{11}=-\frac{\partial \beta}{\partial \rho_{1}}\left(\frac{1-\mu}{\rho_{1}^{\eta}}\right)
$$

A.2 Collinear points: $L_{1}$-type and $L_{2}$-type

In this case

$$
\left[\boldsymbol{\rho}_{1}\right]_{\mathcal{T}}=\left[\begin{array}{lll}
\rho_{1} & 0 & 0
\end{array}\right]^{\mathrm{T}} \quad, \quad\left[\boldsymbol{\rho}_{2}\right]_{\mathcal{T}}=\left[\begin{array}{lll}
\left(\rho_{1}-1\right) & 0 & 0
\end{array}\right]^{\mathrm{T}}
$$


and Eq. (49) provides

$$
\begin{aligned}
& k_{11}=2 \frac{1-\mu}{\rho_{1}^{3}} \pm 2 \frac{\mu}{\left(\rho_{1}-1\right)^{3}}+1-\frac{\eta}{\rho_{1}}\left[\frac{1-\mu}{\rho_{1}^{2}} \pm \frac{\mu}{\left(1-\rho_{1}\right)^{2}}-\rho_{1}+\mu\right] \\
& k_{22}=k_{33}+1=\frac{\mu}{\rho_{1}}\left[1 \pm \frac{1}{\left(1-\rho_{1}\right)^{3}}\right] \\
& k_{12}=k_{21}=k_{23}=k_{32}=k_{13}=k_{31}=0
\end{aligned}
$$

where the sign of the terms containing \pm is + for $L_{2}$-type points and - for $L_{1}$-type points.

\section{A.3 Triangular-type points}

The triangular-type points are characterized by means of the vectors

$$
\left[\boldsymbol{\rho}_{1}\right]_{\mathcal{T}}=\left[\begin{array}{c}
\rho_{1}^{2} / 2 \\
\pm \sqrt{\rho_{1}^{2}-\rho_{1}^{4} / 4} \\
0
\end{array}\right] \quad, \quad\left[\boldsymbol{\rho}_{2}\right]_{\mathcal{T}}=\left[\begin{array}{c}
\rho_{1}^{2} / 2-1 \\
\pm \sqrt{\rho_{1}^{2}-\rho_{1}^{4} / 4} \\
0
\end{array}\right]
$$

Because $\beta$ is given by Eq. (29), from Eq. (49) one obtains

$$
\begin{aligned}
& k_{11}=\frac{1-\mu}{\rho_{1}^{3}}\left(\frac{3 \rho_{1}^{2}}{4}-1\right)+\mu\left[3\left(\frac{\rho_{1}^{2}}{2}-1\right)^{2}-1\right]+1-(1-\mu)\left(\frac{1}{\rho_{1}^{3}}-1\right)\left(\frac{\eta+1}{4} \rho_{1}^{2}-1\right) \\
& k_{22}=\frac{1-\mu}{\rho_{1}^{3}}\left(2-\frac{3 \rho_{1}^{2}}{4}\right)+\mu\left(3 \rho_{1}^{2}-\frac{3 \rho_{1}^{4}}{4}-1\right)+1-(1-\mu)\left(\frac{1}{\rho_{1}^{3}}-1\right)\left[(\eta+1)\left(1-\frac{\rho_{1}^{2}}{4}\right)-1\right] \\
& k_{33}=-1 \\
& k_{12}=k_{21}= \pm \sqrt{\rho_{1}^{2}-\frac{\rho_{1}^{4}}{4}}\left[\frac{3(1-\mu)}{2 \rho_{1}^{3}}+3 \mu\left(\frac{\rho_{1}^{2}}{2}-1\right)-\frac{(1-\mu)(\eta+1)}{2}\left(\frac{1}{\rho_{1}^{3}}-1\right)\right] \\
& k_{13}=k_{31}=k_{23}=k_{32}=0
\end{aligned}
$$

where the sign of the terms containing \pm is + for points with $y>0$ ad - for points with $y<0$.

\section{A.4 Displaced points}

In this last case one has

$$
\left[\boldsymbol{\rho}_{1}\right]_{\mathcal{T}}=\left[\begin{array}{lll}
\rho_{1_{x}} & 0 & \rho_{1_{z}}
\end{array}\right]^{\mathrm{T}} \quad, \quad\left[\boldsymbol{\rho}_{2}\right]_{\mathcal{T}}=\left[\begin{array}{lll}
\rho_{2_{x}} & 0 & \rho_{1_{z}}
\end{array}\right]^{\mathrm{T}}
$$


where

$$
\rho_{1_{x}}=\rho_{2_{x}}+1=\mu\left(1-1 / \rho_{2}^{3}\right) \quad, \quad \rho_{1_{z}}= \pm \sqrt{\rho_{2}^{2}-\left(-\mu / \rho_{2}^{3}+\mu-1\right)^{2}}
$$

Substituting the expression (31) for $\beta$ into Eq. (49) provides:

$$
\begin{aligned}
& k_{11}=\frac{1-\mu}{\rho_{1}^{5}}\left(3 \rho_{1_{x}}^{2}-\rho_{1}^{2}\right)+\frac{\mu}{\rho_{2}^{5}}\left(3 \rho_{2_{x}}^{2}-\rho_{2}^{2}\right)+1-\frac{\left[(\eta+1) \rho_{1_{x}}^{2}-\rho_{1}^{2}\right]}{\rho_{1}^{2}}\left(\frac{1-\mu}{\rho_{1}^{3}}+\frac{\mu}{\rho_{2}^{3}}\right) \\
& k_{22}=1 \\
& k_{33}=\frac{1-\mu}{\rho_{1}^{5}}\left(3 \rho_{1_{z}}^{2}-\rho_{1}^{2}\right)+\frac{\mu}{\rho_{2}^{5}}\left(3 \rho_{2_{z}}^{2}-\rho_{2}^{2}\right)+1-\frac{\left[(\eta+1) \rho_{1_{z}}^{2}-\rho_{1}^{2}\right]}{\rho_{1}^{2}}\left(\frac{1-\mu}{\rho_{1}^{3}}+\frac{\mu}{\rho_{2}^{3}}\right) \\
& k_{13}=k_{31}=\rho_{1_{z}}\left[3 \frac{1-\mu}{\rho_{1}^{5}} \rho_{1_{x}}+\frac{3 \mu \rho_{2_{x}}}{\rho_{2}^{5}}-\frac{(\eta+1) \rho_{1_{x}}}{\rho_{1}^{2}}\left(\frac{1-\mu}{\rho_{1}^{3}}+\frac{\mu}{\rho_{2}^{3}}\right)\right] \\
& k_{12}=k_{21}=k_{23}=k_{32}=0
\end{aligned}
$$

where $\rho_{1}=\sqrt{\rho_{2}^{2}-1+2 \mu\left(1-1 / \rho_{2}^{3}\right)}$ and the sign of the terms containing \pm is + for points with $z>0$ ad - for points with $z<0$.

\section{Acknowledgments}

This research was financed in part by a "Paolo Manes" grant. Alessandro A. Quarta gratefully acknowledges the support of the Manes family.

\section{References}

Abramowitz, M., Stegun, I. A., June 1 1965. Handbook of Mathematical Functions: with Formulas, Graphs, and Mathematical Tables. Dover Publications, p. 17, ISBN: 0486612724.

Baig, S., McInnes, C. R., November-December 2008. Artificial three-body equilibria for hybrid low-thrust propulsion. Journal of Guidance, Control, and Dynamics 31 (6), 1644-1654, doi: 10.2514/1.36125.

Battin, R. H., 1987. An Introduction to the Mathematics and Methods of Astrodynamics. AIAA, New York, pp. 371-381, ISBN: 1563473429 .

Bombardelli, C., Peláez, J., January 2011. On the stability of artificial equilibrium points in the circular restricted three-body problem. Celestial Mechanics and Dynamical Astronomy 109 (1), 13-26, doi: 10.1007/s10569-010-9317-z.

Bookless, J., McInnes, C. R., May 2008. Control of Lagrange point orbits using solar sail propulsion. Acta Astronautica 62, 159-176, doi: 10.1016/j.actaastro.2006.12.051.

Bourke, R. D. Sauer, G. G. J., April 17-19 1972. The effect of solar array degradation on electric propulsion spacecraft performance. In: AIAA 9th Electric Propulsion Conference. Bethesda, MD, USA, paper AIAA $72-444$. 
Brophy, J. R., 3-7 September 2001. NASA's Deep Space 1 ion engine. In: 9th International Conference on Ion Sources. Oakland, California, USA, paper ICIS-01-20.1.

Brophy, J. R., January-March 2003. Advanced ion propulsion systems for affordable deep-space missions. Acta Astronautica 52 (2-6), 309-316, doi: 10.1016/S0094-5765(02)00170-4.

Dandouras, I., Pirard, B., Prado, J. Y., 2004. High performance solar sails for linear trajectories and heliostationary missions. Advances in Space Research 34 (1), 198-203, doi: 10.1016/j.asr.2003.02.055.

Dusek, H. M., September 16-17 1965. Motion in the vicinity of libration points of a generalized restricted three body model. AIAA/ION Astrodynamics Specialist Conference, AIAA, Monterey, California, AIAA Paper 65-682.

Forward, September-October 1991. Statite - a spacecraft that does not orbit. Journal of Spacecraft and Rockets 28 (5), 606-611, doi: $10.2514 / 3.26287$.

Frisbee, R. H., November-December 2003. Advanced space propulsion for the 21st century. Journal of Propulsion and Power 19 (6), 1129-1154, doi: 10.2514/2.6948.

Gong, S., Li, J., BaoYin, H., November 2009. Formation flying solar-sail gravity tractors in displaced orbit for towing near-earth asteroids. Celestial Mechanics and Dynamical Astronomy 105 (1-3), 159-177, doi: 10.1007/s10569-009-9211-8.

Henrici, P., February 23 1988. Applied and Computational Complex Analysis, Power Series Integration Conformal Mapping Location of Zero. Vol. 1. Wiley-Interscience, p. 442, ISBN: 0471608416.

Higuchi, K., Ishimura, K., May 4-7 2009. A survey of space structures research in Japan. In: 50th AIAA/ASME/ASCE/AHS/ASC Structures, Structural Dynamics, and Materials Conference. Palm Springs, California, paper AIAA-2009-2387.

Hunt, M. E., 1993. High efficiency dynamic radioisotope power system for space exploration - a status report. IEE AES System Magazine 8 (12), 18-23.

Janhunen, P., September-October 2004. Electric sail for spacecraft propulsion. Journal of Propulsion and Power 20 (4), 763-764, doi: 10.2514/1.8580.

Janhunen, P., 6-9 July 2009. Status report of the electric sail - a revolutionary near-term propulsion technique in the solar system. Sixth IAA Symposium on Realistic Near-Term Advanced Scientific Space Missions, Aosta, Italy.

Janhunen, P., September 19-24 2010. The electric solar wind sail status report. Vol. 5. European Planetary Science Congress, paper EPSC 2010-297.

Janhunen, P., March-April 2011. Status report of the electric sail in 2009. Acta Astronautica 68 (5-6), 567-570, doi: 10.1016/j.actaastro.2010.02.007 |.

Janhunen, P., Sandroos, A., 2007. Simulation study of solar wind push on a charged wire: basis of solar wind electric sail propulsion. Annales Geophysicae 25 (3), 755-767.

Johnson, L., Whorton, M., Heaton, A., Pinson, R., Laue, G., Adams, C., March-April 2011. NanoSail-D: A solar sail demonstration mission. Acta Astronautica 68 (5-6), 571-575, doi: 10.1016/j.actaastro.2010.02.008.

Kechichian, J. A., October 1995. Optimal low-thrust transfer using variable bounded thrust. Acta Astronautica 36 (7), 357-365, doi: 10.1016/0094-5765(95)00112-3. 
Kunitsyn, A. L., Perezhogin, A. A., November 1978. On the stability of triangular libration points of the photogravitational restricted circular three-body problem. Celestial Mechanics 18, 395-408, doi: 10.1007/BF01230352.

Leipold, M., Kassing, D., Eiden, M., Herbeck, L., june 1999. Solar sails for space exploration - the development and demonstration of critical technologies in partnership. Tech. rep., ESA bullettin 98.

Lichodzeijewski, D., Derbes, B., May 1-4 2006. Vacuum deployment and testing of a 20m solar sail system. In: 47th AIAA/ASME/ASCE/AHS/ASC Structures, Structural Dynamics, and Materials Conference. Newport, Rhode Island, paper AIAA-2006-1705.

Lyngvi, A. E., van den Berg, M. L., Falkner, P., 17 April 2007. Study overview of the interstellar heliopause probe. Tech. Rep. 3 (revision 4), ESA, reference: SCI-A/2006/114/IHP. Available online (cited March 14, 2011) http://esagrid.esa. int/science-e/www/object/index.cfm?fobjectid=40926.

Macdonald, M., Hughes, G., McInnes, C. R., Lyngvi, A., Falkner, P., Atzei, A., September-October 2006. Solar polar orbiter: A solar sail technology reference study. Journal of Spacecraft and Rockets 43 (5), 960-972, doi: 10.2514/1.16408.

Macdonald, M., McInnes, C. R., Hughes, G., May-June 2010. Technology requirements of exploration beyond Neptune by solar sail propulsion. Journal of Spacecraft and Rockets 47 (4), 472-483, doi: 10.2514/1.46657.

McInnes, C. R., 27th April-1st May 1998a. Mission applications for high performance solar sails. 3rd IAA Conference on Low Cost Planetary Missions, California Institute of Technology, Pasadena (CA), IAF-ST-W.1.05.

McInnes, C. R., November-December 1998b. Passive control of displaced solar sail orbits. Journal of Guidance, Control, and Dynamics 21 (6), 975-982, doi: 10.2514/2.4334.

McInnes, C. R., January-February 1999a. Artificial Lagrange points for a partially reflecting flat solar sail. Journal of Guidance, Control, and Dynamics 22 (1), 185-187, doi: 10.2514/2.7627.

McInnes, C. R., 1999b. Solar Sailing: Technology, Dynamics and Mission Applications. Springer-Praxis Series in Space Science and Technology. Springer-Verlag, pp. 38-40, ISBN: 185233102X.

McInnes, C. R., September-October 2003. Orbits in a generalized two-body problem. Journal of Guidance, Control, and Dynamics 26 (5), 743-749, doi: 10.2514/2.5129.

McInnes, C. R., McDonald, A. J. C., Simmons, J. F. L., MacDonald, E. W., March-April 1994. Solar sail parking in restricted three-body systems. Journal of Guidance, Control, and Dynamics 17 (2), 399-406, doi: 10.2514/3.21211.

McKay, R. J., Macdonald, M., Biggs, J., McInnes, C. R., 2011. Survey of highly non-keplerian orbits with low-thrust propulsion. Journal of Guidance, Control and Dynamics, in press .

Mengali, G., Quarta, A. A., November-December 2005. Fuel-optimal, power-limited rendezvous with variable thruster efficiency. Journal of Guidance, Control, and Dynamics 28 (6), 1194-1199, doi: 10.2514/1.12480.

Mengali, G., Quarta, A. A., September-October 2006. Minimagnetospheric plasma propulsion for outer planet missions. Journal of Guidance, Control, and Dynamics 29 (5), 1239-1242, doi: 10.2514/1.21634.

Mengali, G., Quarta, A. A., April-May 2007. Optimal heliostationary missions of high-performance sailcraft. Acta Astronautica 60 (8-9), 676-683, doi: 10.1016/j.actaastro.2006.07.018. 
Mengali, G., Quarta, A. A., May-June 2009a. Escape from elliptic orbit using constant radial thrust. Journal of Guidance, Control, and Dynamics 32 (3), 1018-1022, doi: 10.2514/1.43382.

Mengali, G., Quarta, A. A., November 2009b. Non-keplerian orbits for electric sails. Celestial Mechanics and Dynamical Astronomy 105 (1-3), 179-195, doi: 10.1007/s10569-009-9200-y.

Mengali, G., Quarta, A. A., Janhunen, P., January-February 2008. Electric sail performance analysis. Journal of Spacecraft and Rockets 45 (1), 122-129, doi: 10.2514/1.31769.

Morimoto, M., Yamakawa, H., Uesugi, K., September-October 2007. Artificial equilibrium points in the low-thrust restricted three-body problem. Journal of Guidance, Control and Dynamics 30 (5), 1563-1567, doi: 10.2514/1.26771.

Ozimek, M., Grebow, D., Howell, K., November-December 2009. Design of solar sail trajectories with applications to lunar south pole coverage. Journal of Guidance, Control, and Dynamics 32 (6), 1884-1897, doi: 10.2514/1.41963.

Perezhogin, A. A., September 1976. Stability of the sixth and seventh libration points in the photogravitational restricted circular three-body problem. Soviet Astronomy Letters 2, 174-175 .

Perezhogin, A. A., Tureshbaev, A. T., Jul. 1989. Stability of coplanar libration points in the photo gravitational restricted three-body problem. Soviet Astronomy Letters ASTR. 33 (4), 445-448 .

Prussing, J. E., Coverstone, V. L., May-June 1998. Constant radial thrust acceleration redux. Journal of Guidance, Control, and Dynamics 21 (3), 516-518, doi: 10.2514/2.7609.

Quarta, A. A., Mengali, G., December 2009. Optimal switching strategy for radially accelerated trajectories. Celestial Mechanics and Dynamical Astronomy 105 (4), 361-377, doi: 10.1007/s10569-009-9233-2.

Quarta, A. A., Mengali, G., May-June 2010. Electric sail mission analysis for outer solar system exploration. Journal of Guidance, Control, and Dynamics 33 (3), 740-755, doi: 10.2514/1.47006.

Quarta, A. A., Mengali, G., February 2011a. Analytical results for solar sail optimal missions with modulated radial thrust. Celestial Mechanics and Dynamical Astronomy 109 (2), 147-166, doi: 10.1007/s10569-010-9319-x.

Quarta, A. A., Mengali, G., 2011b. Minimum-time space missions with solar electric propulsion. Aerospace Science and Technology, in press, doi: 10.1016/j.ast.2010.09.003.

Racca, G. D., January 2003. New challenges to trajectory design by the use of electric propulsion and other new means of wandering in the solar system. Celestial Mechanics and Dynamical Astronomy 85 (1), 1-24, doi: 10.1023/A:1021787311087.

Rayman, M. D., Williams, S. N., July-August 2002. Design of the first interplanetary solar electric propulsion mission. Journal of Spacecraft and Rockets 39 (4), 589-595, doi: 10.2514/2.3848.

Richardson, A. J., Warren, J. W., June 1971. Solar array degradation due to meteoroid impacts during extended planetary missions. Journal of Spacecraft and Rockets 8 (6), 681-683, doi: 10.2514/3.59713.

Saleh, J. H., Hastings, D. E., Newman, D. J., March-April 2002. Spacecraft design lifetime. Journal of Spacecraft and Rockets 39 (2), 244-257, doi: 10.2514/2.3806.

Sauer, Jr., C. G., April 25-27 1978. Modeling of thruster and solar array characteristics in the jpl low-thrust trajectory analysis. 13th International Electric Propulsion Conference, San Diego, CA, USA, paper AIAA 78-645. 
Sauer, Jr., C. G., August 15-19 1999. Solar sail trajectories for solar polar and interstellar probe missions. AAS/AIAA Astrodynamics Specialist Conference, Girdwood, Alaska, USA, paper AAS 99-336.

Schuerman, D. W., May 1980. The restricted three-body problem including radiation pressure. Astrophysical Journal, Part 1238 (1), 337-342, doi: 10.1086/157989.

Simmons, J. F. L., McDonald, A. J. C., Brown, J. C., February 1985. The restricted 3-body problem with radiation pressure. Celestial Mechanics 35 (2), 145-187, doi: 10.1007/BF01227667.

Szebehely, V., June 1967. Theory of orbits: the restricted problem of three bodies. Academic Press Inc., p. 250, ISBN: 0126806500

Trask, A. J., Mason, W. J., Coverstone, V. L., May-June 2004. Optimal interplanetary trajectories using constant radial thrust and gravitational assists. Journal of Guidance, Control, and Dynamics 27 (3), 503-506, doi: 10.2514/1.2586.

Vadali, S. R., Nah, R. S., Braden, E., Johnson, Jr., I. L., May-June 2000. Fuel-optimal planar Earth-Mars trajectories using low-thrust exhaust-modulated propulsion. Journal of Guidance, Control, and Dynamics 23 (3), 476-482, doi: $10.2514 / 2.4553$

Vulpetti, G., May 1997. Sailcraft at high speed by orbital angular momentum reversal. Acta Astronautica 40 (10), 733-758, doi: 10.1016/S0094-5765(97)00153-7.

Vulpetti, G., Scaglione, S., January-February 1999. The aurora project: Estimation of the optical sail parameters. Acta Astronautica 44 (2-4), 123-132, doi: 10.1016/S0094-5765(99)00038-7.

Waters, T. J., McInnes, C. R., July 2008. Solar sail dynamics in the three-body problem: Homoclinic paths of points and orbits. International Journal of Non-Linear Mechanics 43 (6), 490-496, doi: 10.1016/j.ijnonlinmec.2008.01.001.

West, J., August 18-21 2008. The lunar polesitter. In: AIAA/AAS Astrodynamics Specialist Conference and Exhibit. Honolulu, Hawaii, paper AIAA-2008-7073.

Williams, S. N., Coverstone-Carroll, V., April-June 1997. Benefits of solar electric propulsion for the next generation of planetary exploration missions. Journal of the Astronautical Sciences 45 (2), 143-159 .

Winglee, R. M., Slough, J., Ziemba, T., Goodson, A., 2000. Mini-magnetospheric plasma propulsion: Tapping the energy of the solar wind for spacecraft propulsion. Journal of Geophysical Research 105 (A9), 21,067-21,077 .

Xu, M., Xu, S., December 2008. Nonlinear dynamical analysis for displaced orbits above a planet. Celestial Mechanics and Dynamical Astronomy 102 (4), 327-353, doi: 10.1007/s10569-008-9171-4.

Zubrin, R. M., Andrew, D. G., March-April 1991. Magnetic sails and interplanetary travel. Journal of Spacecraft and Rockets 28 (2), 197-203, doi: 10.2514/3.26230. 